\title{
Is it possible to model observed active region coronal emission simultaneously in EUV and X-ray filters?
}

\author{
J. Dudík ${ }^{1,2}$, E. Dzifčáková ${ }^{2}$, M. Karlický ${ }^{2}$, and A. Kulinováá, \\ ${ }^{1}$ Department of Astronomy, Physics of the Earth, and Meteorology, Faculty of Mathematics, Physics and Informatics, Comenius \\ University, Mlynská Dolina F2, 84248 Bratislava, Slovak Republic \\ e-mail: dudik@fmph.uniba.sk \\ 2 Astronomical Institute of the Academy of Sciences of the Czech Republic, Fričova 298, 25165 Ondřejov, Czech Republic
}

Received 18 October 2010 / Accepted 4 May 2011

\section{ABSTRACT}

\begin{abstract}
Aims. We investigate the possibility of modeling the active region coronal emission in the EUV and X-ray filters using one, universal, steady heating function, tied to the properties of the magnetic field.

Methods. We employ a simple, static model to compute the temperature and density distributions in the active region corona. The model allows us to explore a wide range of parameters of the heating function. The predicted EUV and X-ray emission in the filters of EIT/SOHO and XRT/Hinode are calculated and compared with observations. Using the combined improved filter-ratio (CIFR) method, a temperature diagnostic is employed to compare the modeled temperature structure of the active region with the temperature structure derived from the observations.

Results. The global properties of the observations are most closely matched for heating functions scaling as $B_{0}^{0.7-0.8} / L_{0}^{0.5}$ that depend on the spatially variable heating scale-length. The modeled X-ray emission originates from locations where large heating scale-lengths are found. However, the majority of the loops observed in the 171 and 195 filters can be modeled only by loops with very short heating scale-lengths. These loops are known to be thermally unstable. We are unable to find a model that both matches the observations in all EUV and X-ray filters, and contains only stable loops. As a result, although our model with a steady heating function can explain some of the emission properties of the 171 and 195 loops, it cannot explain their observed lifetimes. Thus, the model does not lead to a self-consistent solution. The performance of the CIFR method is evaluated and we find that the diagnosed temperature can be approximated with a geometric mean of the emission-measure weighted and maximum temperature along the line of sight. Conclusions. We conclude that if one universal heating function exists, it should be at least partially time-dependent.
\end{abstract}

Key words. Sun: corona - Sun: UV radiation - Sun: X-rays, gamma rays - Sun: activity - hydrodynamics - methods: data analysis

\section{Introduction}

The solar corona is a rather complex environment. Space-borne observations, made mostly in the extreme ultraviolet (EUV) and $\mathrm{X}$-ray spectral domain, have revealed that the corona is highly structured, with the emission concentrated into spatially confined, individual structures that are sometimes closely packed together. The presence of a magnetic field, a high degree of ionization of the low- $\beta$ coronal plasma, and the frozen-in condition ensures that these structures are thermally isolated from their surroundings. The most basic structures are coronal loops, which are thin, elongated, arch-like, bent cylinders delineating magnetic field lines, rooted in the solar chromosphere on one or both ends. Coronal loops commonly occur above photospheric magnetic flux concentrations, i.e., in active regions, with the hot X-ray loops (>2 MK; e.g., Klimchuk et al. 2010) constituting the active region core, and the warm $(\sim 1 \mathrm{MK})$ EUV loops located on the periphery. Apart from the coronal loops, coronal emission can originate from bright points located above small bipolar photospheric regions, and in the EUV spectral domain from the moss (e.g., Peres et al. 1994; Berger et al. 1999; Fletcher \& De Pontieu 1999; Schrijver et al. 1999; Martens et al. 2000), i.e., upper transition region of hot, high-pressure loops.

The very existence of the solar corona implies that there is of some sort of heating mechanism or mechanisms. Despite decades of theoretical and observational development, the coronal heating mechanism remains elusive. This is commonly referred to as the coronal heating problem (see e.g. Aschwanden 2005; Klimchuk 2006, for a review). The spatial and temporal properties of coronal emission and thus the spatial and temporal properties of temperature and density are linked to the properties of the heating function. Because of this, constraints on the coronal heating function can be derived. This is commonly done in two ways: either by directly analyzing the observations (e.g., Porter \& Klimchuk 1995; Aschwanden et al. 1999, 2000, 2008; Ugarte-Urra et al. 2009; Reale et al. 2009a,b; Warren et al. 2010, to name just a few), or by forward modeling of the observed emission distribution under a set of assumptions including energy equilibrium (e.g., Schrijver \& Aschwanden 2002; Schrijver et al. 2004; Mok et al. 2005; Warren \& Winebarger 2006, 2007; Mok et al. 2008; Gontikakis et al. 2008; Winebarger et al. 2008; Lundquist et al. 2008a,b). While the active region core appears to be consistent with steady heating (Antiochos et al. 2003; Warren et al. 2010), the majority of the EUV loops are not (Aschwanden et al. 2001; Winebarger et al. 2003). However, they can be explained by dynamical heating (e.g., Susino et al. 2010; Klimchuk et al. 2010, and references therein). Apart from this, the connection of the heating function to the magnetic structure of the active region corona remains unclear, although the results of Schrijver \& Aschwanden (2002) and Mok et al. (2008) suggest that heating is locally dependent on the magnetic field.

Furthermore, coronal loops exhibit roughly constant diameter (e.g., Klimchuk 2000; Watko \& Klimchuk 2000; López Fuentes et al. 2006, 2008). This is in contrast to the roughly 
potential-like shape of the magnetic field constituting these coronal loops, since flux-tubes of the potential field are expanding with height. The effect of loop expansion is likely to play a significant role in the emission distribution in the corona and also in the transition region, since the variations in the emitting volume depend on the shape of the flux-tube. By taking into account that the loop expansion is inversely proportional to the magnetic field, models have been found to match the observations (Schrijver et al. 2004; Brooks et al. 2008) and also to explain the observed contrast in the EUV structures (DeForest 2007).

In summary, despite two decades of continuous observations of active region corona with instruments such as Yokhoh, $\mathrm{SOHO}$, TRACE, Hinode, STEREO, and now SDO, it is still unclear whether the observations can be modeled with one single, universal heating function. We note especially that some previous forward models of active region corona (Warren \& Winebarger 2006, 2007) have not been successful in reproducing both the $\mathrm{X}$-ray and EUV filter observations, or did not attempt to model the EUV observations at all (Lundquist et al. 2008a,b). The question of whether these observations can be modeled simultaneously is addressed in this paper under the asumption of steady heating. We expand some of the previous works by tying the heating function to the properties of the magnetic field, described by the loop expansion factor and the heating scale-height, and also by including the phase average of the thermally unstable loops. The description of the model, including the parametrization of the heating function, is summarized in Sect. 2. Section 3 deals with observations of the active region NOAA 10963 that are modeled in this paper. Our results are given in Sect. 4, and discussed in Sect. 5. Section 6 summarizes the conclusions drawn in this paper, and gives a possible answer to the question posed in the title.

\section{Model construction}

The aim of this section is to construct a model of the active region coronal emission. To do this, we employ an analytical approximative method using potential field extrapolations and scaling laws for coronal loops. This approach allows us convert the assumed magnetic field model to the temperature and density distributions in the active region corona. The analytical model is used because constructing a 3D MHD model of the active region corona is a computationally challenging task. Reducing the problem using analytical approximations allows us to explore a large parameter range for the assumed function of the coronal heating, which is one of the purposes of this paper.

\subsection{Magnetic field extrapolation}

To compute the model of the magnetic field $\boldsymbol{B}$ of the active region, the potential approximation is assumed

$$
\boldsymbol{\nabla} \times \boldsymbol{B}=\mathbf{0} .
$$

The potential approximation is the lowest approximation possible, since it assumes that the electric current $\boldsymbol{j}$ vanishes everywhere in the active region.

We note that the validity of the potential approximation is questionable. For instance, Sandman et al. (2009) and Aschwanden \& Sandman (2010) found considerable misalignment between the magnetic field obtained from the potential approximation in comparison to the shapes of the coronal loops derived from the STEREO/EUVI observations (Wülser et al. 2004). However, López Fuentes et al. (2006) showed that linear force-free fields can also be quite poor approximations of the real magnetic field in active regions. Even non-linear force-free field models, which are solutions of the equation $\boldsymbol{\nabla} \times \boldsymbol{B}=\alpha \boldsymbol{B}$ with $\alpha \neq$ const., can produce misalignments with the real coronal loops. These misalignments are comparable to the misalignments of the potential field models (DeRosa et al. 2009).

Lundquist et al. (2008a) found that employing the non-linear force-free field leads only to a marginal improvement in the calculated emission model. Gontikakis et al. (2008) concluded that $\alpha \approx 0$ for a bundle of 360 coronal loops, even though $\alpha$ can vary from loop to loop. In their model, the currents along each loop were generated by differences in electric potential between footpoints caused by the random displacements of footpoints in turn caused by random motions of photospheric plasma. Gudiksen \& Nordlund $(2005 a, b)$ found that the coronal field is close to the potential one for regions without strong photospheric shear.

For these reasons, we retained the potential approximation of the magnetic field, even if part of the X-ray loops in the core of the active region modeled in this paper (Sect. 3.1.1) do not correspond to the potential approximation. We utilized the magnetic field extrapolation method of Alissandrakis (1981) and Gary (1989) based on Fourier transformations. This method requires only the vertical component of the magnetic field, $B_{z}$, as the boundary condition. This component can be obtained from the longitudinal component as commonly observed by e.g. the MDI/SOHO instrument (Scherrer et al. 1995), if the observed portion of the photosphere lies near the disk center.

\subsection{Temperature and density distributions}

To compute the distributions of temperature and density in the active region corona, we employed the scaling laws derived by Dudík et al. (2009a), with a few modifications. The computational scheme is outlined below.

We assume that a coronal loop is a single-strand structure lying along a magnetic field line, i.e., the loop geometry is given by the magnetic field. The loop is anchored in the photosphere on both ends and its cross-section expands with height in the corona because of the expansion of the magnetic field. The temperature and density structure are the result of the stationary energy balance between coronal heating $E_{\mathrm{H}}$, radiative losses $E_{\mathrm{R}}$, and the divergence of the thermal conductive flux $F_{\mathrm{C}}$

$-E_{\mathrm{R}}+E_{\mathrm{H}}-\boldsymbol{\nabla} \boldsymbol{F}_{\mathrm{C}}=0$

where we assume non-zero thermal conductivity only along the magnetic field (Spitzer 1962)

$F_{\mathrm{C}}(s)=\kappa_{0} T^{5 / 2} \frac{\mathrm{d} T}{\mathrm{~d} s}$

where $\kappa_{0} \doteq 9.2 \times 10^{-12} \mathrm{~W} \mathrm{~m}^{-1} \mathrm{~K}^{-7 / 2}$ is the Spitzer thermal conduction coefficient, $0 \leq s \leq L$ represents the coordinate along the field line, and $T=T(s)$. The position $s=L$ represents the position of the loop top.

The heating term is assumed to be time-independent, i.e., steady, and exponentially decreasing along the coronal section of the loop (Sect. 2.3)

$E_{\mathrm{H}}\left(s \geq s_{0}\right)=E_{\mathrm{H} 0} \exp \left(-\frac{s-s_{0}}{s_{\mathrm{H}}}\right)$,

where $s_{0}$ denotes the position of the footpoint in the upper chromosphere and $s_{\mathrm{H}}$ is the heating scale-length. 
J. Dudík et al.: Is it possible to model observed active region coronal emission simultaneously in EUV and X-ray filters?

The radiative-loss term is assumed to have a power-law dependence on the temperature $T$ characterized by the power-law index $\sigma$ and multiplication constant $\chi$

$E_{\mathrm{R}}(\chi, \sigma)=\chi n_{\mathrm{e}}^{2} T^{\sigma}$.

The electron density $n_{\mathrm{e}}$ entering Eq. (5) is linked to the plasma pressure and temperature by the equation of state

$p(s)=q n_{\mathrm{e}}(s) k_{\mathrm{B}} T(s)$,

where $q \approx 2$ is the number of particles per free electron and $k_{\mathrm{B}} \approx$ $1.38 \times 10^{-23} \mathrm{~J} \mathrm{~K}^{-1}$ is the Boltzmann constant. We assume that the coronal plasma is in hydrostatic equilibrium along a given loop. The pressure stratification is then given by following relation (Aschwanden \& Schrijver 2002)

$p(s)=p_{0}\left(s_{0}\right) \exp \left(-\frac{z(s)-z_{0}\left(s_{0}\right)}{\lambda_{p}(s)\left(1+z(s) / R_{\odot}\right) q_{\lambda}}\right)$,

which holds for non-isothermal plasma along the loop. In this relation, the height profile $z=z(s)$ of the loop is given by loop geometry, $\lambda_{p}(s)=k_{\mathrm{B}} T(s) / \mu m_{\mathrm{H}} g_{\odot}$ is the pressure scale-height, $\mu$ is the mean particle weight, $m_{\mathrm{H}}$ is the mass of the hydrogen atom, and $q_{\lambda}$ is the correction factor for the non-isothermal loops

$q_{\lambda}\left(L, s_{\mathrm{H}}, T_{1}\right)=c_{0}\left(T_{1}\right)+c_{1}\left(T_{1}\right)\left(\frac{L_{0}}{s_{\mathrm{H}}}\right)^{c_{2}\left(T_{1}\right)}$,

given by Aschwanden \& Schrijver (2002). Here we use $L_{0}=$ $L-s_{0}$ instead of $L$. The coefficients $c_{i}$ are functions of the temperature $T_{1}$ at the loop top (apex) and are given in Table 1 .

The apex temperature $T_{1}$ and base pressure $p_{0}$ are tied to the loop parameters by the scaling laws (Dudík et al. 2009a)

$$
\begin{aligned}
T_{1}= & \left(\frac{4}{7 \kappa_{0}}\right)^{2 / 7} I(\sigma, P)^{-4 / 7} E_{\mathrm{H} 0}^{2 / 7} L_{0}^{4 / 7} \\
& \times \exp \left(-\frac{2}{7} \beta_{2} \frac{z_{1}-z_{0}}{\lambda_{p}\left(z_{1}\right)\left(1+z_{1} / R_{\odot}\right) q_{\lambda}}\right) \exp \left(-\frac{2}{7} \gamma_{2} \frac{L_{0}}{s_{\mathrm{H}}}\right), \\
p_{0}= & L_{0}^{-1} T_{1}^{(11-2 \sigma) / 4}\left(\frac{3+2 \sigma}{7} \frac{q^{2} k_{\mathrm{B}}^{2}}{\chi} \frac{7 \kappa_{0}}{4} I(\sigma, P)^{2} P^{\prime}\right)^{1 / 2} \\
& \times \exp \left(-3 \beta_{1} \frac{z_{1}-z_{0}}{\lambda_{p}\left(z_{1}\right)\left(1+z_{1} / R_{\odot}\right) q_{\lambda}}\right) \exp \left(-3 \gamma_{1} \frac{L_{0}}{s_{\mathrm{H}}}\right),
\end{aligned}
$$

where $z_{1}=z(s=L)$ is the height of the loop top. These scaling laws are the first and second integral of the energy balance (Eq. (2)) under the assumptions of a symmetric loop with monotonically increasing temperature profile $T(s)$ and vanishing thermal conduction across the loop top and footpoints (Dudík et al. 2009a). Their comparison to the earlier derived scaling laws of Rosner et al. (1978), Serio et al. (1981), and Aschwanden \& Schrijver (2002) is also given by Dudík et al. (2009a).

The presence of the parameters $P, P^{\prime}, \beta_{1}, \beta_{2}, \gamma_{1}$, and $\gamma_{2}$ are caused by the use of the mean-value theorem during the derivation of the scaling laws. Their values are found by approximating the numerical solutions of the energy balance equation given in Eq. (2) with the scaling laws. The numerical solutions are constructed using the hydro package (Aschwanden \& Schrijver 2002) of the SolarSoft environment in the regime $L_{0} / s_{\mathrm{H}} \leq 3$ corresponding to stable loops (Sect. 2.4). During their construction, a radiative-loss function with $\chi=10^{-31.81} \mathrm{~W} \mathrm{~m}^{3} \mathrm{~K}^{1 / 2}$ and $\sigma=1 / 2$ (Kuin \& Martens 1982) together with a semi-circular loop geometry above the height of the chromosphere $z_{0}=3 \mathrm{Mm}$ were assumed. We set the parameters $P$ and $P^{\prime}$ to be equal
Table 1. Coefficients for the $q_{\lambda}$ function (Eq. (8)) and the scaling laws (Eqs. (9) and (10)).

\begin{tabular}{ccccc}
\hline \hline$T_{1}$ & $1 \mathrm{MK}$ & $3 \mathrm{MK}$ & $5 \mathrm{MK}$ & $10 \mathrm{MK}$ \\
\hline$c_{0}$ & +0.6995 & +0.6889 & +0.6848 & +0.6896 \\
$c_{1}$ & +0.0137 & +0.0151 & +0.0173 & +0.0218 \\
$c_{2}$ & +1.1113 & +1.1444 & +1.1358 & +1.1250 \\
\hline$\beta_{1}$ & -0.0163 & +0.0043 & +0.0193 & +0.0473 \\
$\gamma_{1}$ & -0.0779 & -0.0907 & -0.0941 & -0.0703 \\
\hline$\beta_{2}$ & -0.1217 & -0.3734 & -0.5171 & -0.6410 \\
$\gamma_{2}$ & +0.6401 & +0.7624 & +0.7989 & +0.7562 \\
\hline
\end{tabular}

to 1 and compute the best-fit parameters $\beta_{1}, \beta_{2}, \gamma_{1}$, and $\gamma_{2}$ by the Levenberg-Marquardt algorithm. Their values are listed in Table 1 . The relative error of the scaling laws obtained in the described manner is lower than $20 \%$ and $10 \%$, respectively, which is an improvement in the values found for variable $P$ and $P^{\prime}$ by Dudík et al. (2009a).

The temperature profile $T(s)$ is closely approximated by the generalized elliptical function (Aschwanden \& Schrijver 2002)

$T(s)=T_{1}\left(1-S^{a\left(T_{1}\right)}\right)^{b\left(T_{1}\right)}$,

where $S=(L-s) / L_{0}$, and $a\left(T_{1}\right)$ and $b\left(T_{1}\right)$ are given by expressions similar to that of Eq. (8). Values of $a_{i}\left(T_{1}\right)$ and $b_{i}\left(T_{1}\right)$ are given in Table 1 of Aschwanden \& Schrijver (2002). The above relation is valid for $L_{0} / s_{\mathrm{H}} \leq 3$. For higher values of this ratio, up to 25, we can apply the correction (Aschwanden \& Schrijver 2002):

$T(s)=T_{1}\left(1-S^{a\left(T_{1}\right)}\right)^{b\left(T_{1}\right)}\left(1+\frac{1}{2} \log _{10}\left(\frac{L_{0}}{S_{\mathrm{H}}}\right)(1-S) S^{5}\right)$.

For a given $T_{1}$, the relative error of these temperature profiles is less than 1\% (Aschwanden \& Schrijver 2002). In general, the corrected $T(s)$ profile does not have its maximum at the loop top, but at some position $s_{\max }$ along the loop. The value $T_{\max }$ is $\leq 1.05 T_{1}$ (Aschwanden \& Schrijver 2002, Fig. 9 therein). For such $T(s)$, the scaling laws in Eqs. (9) and (10) cannot be derived, because $T(s)$ is not monotonic. However, the small departure of the $T_{\max }$ from $T_{1}$ would allow for an approximation of the temperature profile by constant for $s \geq s_{\max }$. Then, the scaling laws can be employed together with the corrected $T(s)$ profile given in Eq. (12).

We compute the temperature and density distributions $T(x, y, z)$ and $n_{\mathrm{e}}(x, y, z)$ at each voxel (volume element) of the computational box. The lower boundary $z=0$ of the computational box corresponds to a portion of the photosphere represented by the MDI magnetogram (Fig. 1), with the voxel size $\Delta x=\Delta y=\Delta z=1.43 \mathrm{Mm}$ corresponding to the resolution of the MDI instrument.

For each voxel $(x, y, z)$, the field line passing through its center $(x+1 / 2, y+1 / 2, z+1 / 2)$ is traced. For a given field line, and thus a given point, the parameters $s, s_{0}, L_{0}, E_{\mathrm{H} 0}$, and $s_{\mathrm{H}}$ (Sect. 2.3) are determined. These parameters are then used to compute the $T_{1}$ and $p_{0}$ distributions using Eqs. (9) and (10). The effects of non-circular loop geometry are neglected, because they lead only to small changes in $T_{1}$ and $p_{0}$ (Dudík et al. 2009a). Since the expression in Eq. (9) for $T_{1}$ depends on $\lambda_{p}\left(z_{1}\right)$, which itself depends on $T_{1}$, the value of $T_{1}$ is computed iteratively. For convergence reasons, the $\lambda_{p}$ is averaged over two iteration steps. Finally, $T$ and $n_{\mathrm{e}}$ are determined using Eqs. (11), (12), (7), and (6) in this order. The obtained values of $T$ and $n_{\mathrm{e}}$ at the given point $(x+1 / 2, y+1 / 2, z+1 / 2)$ then represents the $T$ and $n_{\mathrm{e}}$ in the entire voxel $(x, y, z)$. 
With a sufficiently high magnetogram resolution, this technique would allow us to capture small spatial variations in $T$ and $n_{\mathrm{e}}$ that could correspond to loop substructuring. However, the pixel size of the MDI instrument is rather large, thus the achieved resolution of $T$ and $n_{\mathrm{e}}$ is unable to resolve any substructures. Nevertheless, the derived temperature and density distributions are directly linked to the properties of the magnetic field (Sect. 2.3).

Another advantage of computating $T$ and $n_{\mathrm{e}}$ in this manner is that there is no pre-selection of field lines along which the emission is computed, which had been done by other authors (e.g., Schrijver et al. 2004; Warren \& Winebarger 2006, 2007; Lundquist et al. 2008a,b; Gontikakis et al. 2008; Brooks et al. 2008). It has also the advantage of uniformly spaced voxels, i.e., there is no danger of under-representation of any given emitting sub-volume of the box because of the low number of field lines passing through it. Any apparent structures on the synthetic images (Sect. 4) must then be due to truly loop-like distributions of temperature and density in the computational box. The conversion of the obtained distributions of $T$ and $n_{\mathrm{e}}$ to the synthetic emission using filter responses is described in Sect. 3.2.

\subsection{The heating term}

To study the effects of various heating functions on the resulting emission, we parametrized the base heating rate $E_{\mathrm{H} 0}$ as a time-independent, power-law function depending on the magnetic field at the footpoint $B_{0}$ and the half-length of the coronal portion of the loop $L_{0}$. The heating function given in Eq. (4) is then

$E_{\mathrm{H} 0} \exp \left(-\frac{s-s_{0}}{s_{\mathrm{H}}}\right)=C_{\mathrm{H} 0}\left(\frac{B_{0}}{B_{\text {ref }}}\right)^{\rho}\left(\frac{L_{\mathrm{ref}}}{L_{0}}\right)^{\tau} \exp \left(-\frac{s-s_{0}}{s_{\mathrm{H}}}\right)$,

where $B_{\text {ref }}=100 \mathrm{G}$ and $L_{\text {ref }}=100 \mathrm{Mm}$ are pre-set reference values so that the $C_{\mathrm{H} 0}\left[\mathrm{~W} \mathrm{~m}^{-3}\right]$ is the base heating rate of a loop with $L_{0}=L_{\text {ref }}$ and $B_{0}=B_{\text {ref. }}$ The $C_{\mathrm{H} 0}$ is treated as a free parameter together with $\rho$ and $\tau$. Such or similar parametrizations of the base heating rate were used by e.g. Schrijver et al. (2004), Warren \& Winebarger (2006, 2007), Lundquist et al. (2008a,b), and Brooks et al. (2008). However, these authors used a priori fixed value of $C_{\mathrm{H} 0}$, i.e., a fixed scaling for temperature. Treating $C_{\mathrm{HO}}$ as a free parameter enables us to adjust the modeled temperature distribution to the temperature distribution derived from the observations (Sect. 3.3).

The heating scale-length $s_{\mathrm{H}}$ is computed as the distance along the loop strand between points where $B^{\rho}$ decreases from $B_{0}^{\rho}$ to $B_{0}^{\rho} /$ e. In practice, we determine $B_{0}$ in the photosphere rather than in the upper chromosphere - transition-region boundary at the height $z_{0}$. This is because the $B_{0 z}$ at the photosphere is directly measured and not derived from the extrapolation method. Since the scaling laws require symmetric heating at both footpoints, the actual value of $B_{0}$ is computed as the average of the magnetic field at footpoints in positive and negative polarity

$B_{0}=\left(B_{0 \mathrm{P}}+B_{0 \mathrm{~N}}\right) / 2$

and a similar relation applies to the computation of $s_{\mathrm{H}}$.

Since the flux-tubes in a potential field expand with height, the loop expansion factor $\Gamma$ is determined as the ratio of the strand cross-section at the apex to the cross-section at the footpoint, $A_{1} / A_{0}$. Flux conservation then leads to the simple expression

$\Gamma=B_{0} / B_{1}$,

where $B_{1}$ is the magnetic field at the apex of the strand.
Aschwanden \& Schrijver (2002) concluded that the effect $\Gamma$ on numerical solutions for coronal loops is similar to the effect of shortening the $s_{\mathrm{H}}$. Using this result, they derived the following correction

$s_{\mathrm{H}}^{\Gamma}=\frac{s_{\mathrm{H}}}{\sqrt{1+(\Gamma-1) s_{\mathrm{H}} / L}}$,

which is valid for $s_{\mathrm{H}} \leq L$. For larger $s_{\mathrm{H}}$, the relation is $s_{\mathrm{H}}^{\Gamma}=$ $L / \sqrt{\Gamma-1}$. We do not use this second correction, since the majority of coronal loops in the model have $s_{\mathrm{H}}<L$ and using the correction given by Eq. (16) introduces an error of within a factor of two even for near-uniformly heated loops. Corrected scale-lengths $S_{\mathrm{H}}^{\Gamma}$ are then used throughout the Eqs. (8)-(12) up to values of $L_{0} / s_{\mathrm{H}}^{\Gamma} \leq 25$. Loops with shorter scale-lengths are discarded as well as open loops, for which the $L_{0}$ cannot be correctly determined.

In practice, one cannot expect the heating to be symmetric at both ends of the loop. In the particular case of AR 10963 studied in this paper (Sect. 3), the parameters $B_{0 \mathrm{P}}, B_{0 \mathrm{~N}}, s_{\mathrm{HP}}$, and $s_{\mathrm{HN}}$ can differ by a factor of ten or more for the two footpoints, even if the total heating $E_{\mathrm{H} 0} s_{\mathrm{H}}^{\Gamma}\left(1-\exp \left(-L_{0} / s_{\mathrm{H}}^{\Gamma}\right)\right)$ along the two halves of the loop rarely differ by more than a factor of 2.5. Such loops are anchored in a positive polarity plage with $B_{0 \mathrm{P}} \approx 200 \mathrm{G}$ on one end and in the negative-polarity sunspot with $B_{0 \mathrm{~N}} \geq 2000 \mathrm{G}$ at the other end. While the $B$ increases slightly with height near the plage footpoint, it decreases rapidly near the sunspot footpoint. There is no stationary hydrodynamic solution for such loops (Lundquist et al. 2008a). In our model, these loops have extremely large $\Gamma$, on the order of $10^{3}-10^{4}$. Thus, the corrected $s_{\mathrm{H}}^{\Gamma}$ fall well below the limit of $L_{0} / s_{\mathrm{H}}^{\Gamma} \leq 25$ and these loops are discarded.

\subsection{Coronal loop stability and the scaling laws}

Very short heating scale-lengths $s_{\mathrm{H}}^{\Gamma}$ can lead to solutions of the energy balance equation given in Eq. (2) that are not stable (e.g., Serio et al. 1981; Aschwanden et al. 2001; Winebarger et al. 2003; Müller et al. 2003, 2004, 2005; Karpen et al. 2005, 2006). In principle, there are two types of instabilities that must be considered: a Rayleigh-Taylor instability occurs if $\boldsymbol{\nabla} n_{\mathrm{e}} \cdot \boldsymbol{g}<0$, and thermal instability occurs if the heating and the thermal conduction near the loop apex are insufficient to balance the radiative losses, leading to the formation of a condensation and condensation-evaporation cycle for the coronal loop (Müller et al. 2003, 2004, 2005).

Aschwanden et al. (2001) found following approximative criterion for the existence of stably stratified coronal loops, that

$s_{\mathrm{H}, \mathrm{Mm}}^{\Gamma} \gtrsim \sqrt{L_{0, \mathrm{Mm}}}$,

where the $s_{\mathrm{H}, \mathrm{Mm}}^{\Gamma}$ and $L_{0, \mathrm{Mm}}$ are expressed in units of $\mathrm{Mm}$. Winebarger et al. (2003) derived the criterion for coronal loops with temperature maximum at the loop apex

$s_{\mathrm{H}, \mathrm{Mm}}^{\Gamma} \gtrsim A(T) L_{0, \mathrm{Mm}}^{\delta(T)}$,

where the parameters $\delta(T) \approx 0.75-0.9$ and $A(T)$ are given in their Table 3. In the region bounded by Eqs. (17) and (18), stable solutions with temperature maximum away from the loop apex (temperature reversals) exist (e.g., Müller et al. 2004, 2005, Figs. 2 and 1 therein, respectively). The temperature profiles of such loops are similar to the profiles given by Eq. (12). Müller et al. (2003, 2004, 2005) numerically studied three loops 
J. Dudík et al.: Is it possible to model observed active region coronal emission simultaneously in EUV and X-ray filters?

with different lengths. The shortest heating scale-lengths for which stable solutions exist follow the stability criterion of Aschwanden et al. (2001) quite closely. According to the results of Müller et al. (2003, 2004, 2005) and also e.g. Klimchuk et al. (2010), loops with shorter scale-lengths undergo the condensation-evaporation cycle during which the loop initially rapidly heats up and fills with plasma because of the chromospheric evaporation. The condensation and subsequent draining caused by the thermal instability then develops because of insufficient heating near the apex. The cycle is periodic with periods of up to several hours or tens of hours. Despite this, Kuin \& Martens (1982) report that the emission measure averaged over many loops with evenly distributed phases is identical to that of a static loop of slightly higher temperature.

The scaling laws given in Eqs. (9) and (10) with the values of the parameters listed in Table 1 are strictly valid only in the range of $L_{0} / s_{\mathrm{H}}^{\Gamma} \leq 3$ where the numerical solutions are found using the hydro package. The numerical solutions for $L_{0} / s_{\mathrm{H}}>3$ are extremely difficult to find for convergence reasons. Because of this, we extrapolate the scaling laws to obtain temperature and density distributions for larger values of the $L_{0} / s_{\mathrm{H}}^{\Gamma}$ ratio, i.e., shorter heating scale-lengths $s_{\mathrm{H}}^{\Gamma}$, which occur in either a strong or rapidly expanding magnetic field. This approach is similar to the one used by Schrijver \& Aschwanden (2002). These authors used the scaling laws of Serio et al. (1981) to model the solar and stellar coronae, but they excluded loops with heating scalelengths shorter than the limit given by the criterion in Eq. (17).

If the numerical coverage of the $L_{0} / s_{\mathrm{H}}^{\Gamma}>3$ region were sufficient, then the validity of our extrapolated scaling laws could be checked, possibly with modifications of the parameters $\beta_{1,2}$ and $\gamma_{1,2}$ to appropriately describe solutions in this region including the average of the cyclic ones.

\section{Observations and their interpretation}

We now present the observations of a particular active region along with the analysis of these observations. The need for observations comes from the simple fact that if the properties of the coronal heating function are to be discerned, the resulting emission model must match the observations. Because the emission model assumes steady heating, static energy and pressure equilibrium, and a potential field approximation, the chosen active region has to be non-flaring, at least roughly potential, and contain loops of vastly different half-lengths (e.g., peripheral loops and a bright point) to constrain the value of $\tau$.

\subsection{Active region NOAA 10963}

The active region NOAA 10963 appeared on the eastern solar limb on 2007 July 8, crossed the central meridian on July 13 and disappeared on the western limb on July 20. It produced 14 C-class flares during the first three days, followed by nonflaring period. The active region was bipolar, with more than one inversion line present until July 12 ( $\beta \gamma$ configuration), and bipolar ( $\beta$ configuration) from July 13 .

The observations used in this paper were obtained by various space-borne imaging instruments on July 13, around 19:00 UT. The observations are summarized in Table 1 and discussed in detail in the following subsections.

The active region, according to the MDI/SOHO magnetogram obtained at 19:12 UT (Fig. 1), consists of a leading negative-polarity spot, a small negative-polarity plage located to the south of the spot, and two smaller positive-polarity spots to
Table 2. Summary of employed AR NOAA 10963 observations.

\begin{tabular}{|c|c|c|c|}
\hline Instrument & Filter & $\begin{array}{c}\text { Time of observations } \\
\text { [UT] }\end{array}$ & $\begin{array}{c}\text { Exposition } \\
{[\mathrm{s}]}\end{array}$ \\
\hline MDI/SOHO & & $19: 12: 02$ & \\
\hline EIT/SOHO & $171 \mathrm{Al}+1$ & $19: 00: 13$ & 12.59 \\
\hline EIT/SOHO & $195 \mathrm{Al}+1$ & $19: 13: 45$ & 12.99 \\
\hline EIT/SOHO & $284 \mathrm{Al}+1$ & 19:06:17 & 122.59 \\
\hline XRT/Hinode & Al-poly/Open & $\begin{array}{l}18: 19: 44,18: 33: 26, \\
19: 16: 35,19: 30: 16, \\
19: 44: 56,19: 58: 39\end{array}$ & $\begin{array}{l}0.36,0.51, \\
0.51,0.51 \\
0.51,0.51\end{array}$ \\
\hline XRT/Hinode & C-poly/Open & $\begin{array}{l}18: 18: 02,18: 31: 46, \\
19: 14: 53,19: 28: 37, \\
19: 43: 14,19: 57: 00\end{array}$ & $\begin{array}{l}.03,1.03 \\
1.03,1.03 \\
1.03,1.03\end{array}$ \\
\hline XRT/Hinode & Open/Ti-poly & $\begin{array}{l}18: 20: 21,18: 34: 03, \\
19: 17: 12,19: 30: 54, \\
19: 45: 33,19: 59: 16\end{array}$ & $\begin{array}{l}.03,1.03 \\
1.03,1.03 \\
1.03,1.03\end{array}$ \\
\hline XRT/Hinode & Be-thin/Open & $\begin{array}{l}18: 19: 12,18: 32: 44, \\
19: 15: 58,19: 29: 34, \\
19: 44: 18,19: 57: 57\end{array}$ & $\begin{array}{l}2.05,2.90 \\
2.90,2.90 \\
2.90,2.90\end{array}$ \\
\hline XRT/Hinode & Be-med/Open & $\begin{array}{l}18: 18: 34,18: 32: 15 \\
19: 15: 25,19: 29: 05 \\
19: 43: 45,19: 57: 27\end{array}$ & $\begin{array}{l}16.39,16.39, \\
16.39,16.39, \\
16.39,16.39\end{array}$ \\
\hline TRACE & 171 & $16: 00: 45-22: 59: 38$ & $55.11-65.34$ \\
\hline XRT/Hinode & C-poly/Open ${ }^{a}$ & $16: 03: 59-22: 59: 45$ & $0.51-1.03$ \\
\hline
\end{tabular}

Notes. ${ }^{a}$ Data used for construction of lightcurves in Fig. 2.

both the east and west sides of a positive-polarity plage (Fig. 1). A secondary bipole (bright point) located to the north of the active region polarities started emerging around 9:30 UT.

\subsubsection{EIT/SOHO and XRT/Hinode observations}

The EUV and X-ray emission of the active region was observed in all four EUV filters by the EUV Imaging Telescope (EIT, Delaboudinière et al. 1995) onboard the SOHO spacecraft (Domingo et al. 1995), and in several X-ray filters and filter combinations by the X-ray telescope (XRT, Golub et al. 2007; Kano et al. 2008) onboard the Hinode spacecraft (Kosugi et al. 2007).

EIT/SOHO imaged the full Sun in all filters every four hours. In this work, we use observations in filters EIT 171, EIT 195, and EIT 284 (Fig. 1) made shortly after 19:00 UT (Table 2).

From the available XRT/Hinode observations, we use observations in five filters, Al-poly/Open, C-poly/Open, Open/Tipoly, Be-thin/Open, and Be-med/Open. In addition to these observations, XRT/Hinode observed the active region using Open/Al-thick, Al-poly/Ti-poly, and C-poly/Al-thick filters. Observations in the Al-thick filter are quite noisy and therefore unusable, while observations in Al-poly/Ti-poly filter combination are not required, since observations in each filter are already available. For simplicity, the "Open" word will be omitted from now on.

The data were calibrated using standard procedures implemented in the SolarSoft environment running under IDL (Freeland \& Handy 1998), namely the eit_prep and $x r t \_$prep routines, which include the exposure normalization and removal of cosmic rays. The calibrated EIT data are quite noisy, probably because of the contamination of the CCD (Defise et al. 1997). To reduce the noise, EIT data are smoothed by the $3 \times 3$ boxcar. The signal in XRT filters were improved by averaging over a time period of 18:00-20:00 UT, i.e., the XRT data used in this paper and displayed in Fig. 1 are the averages of six individual observations (Table 2). The averaging is justified, because the signal observed by XRT varied little with time (Fig. 2 top). 

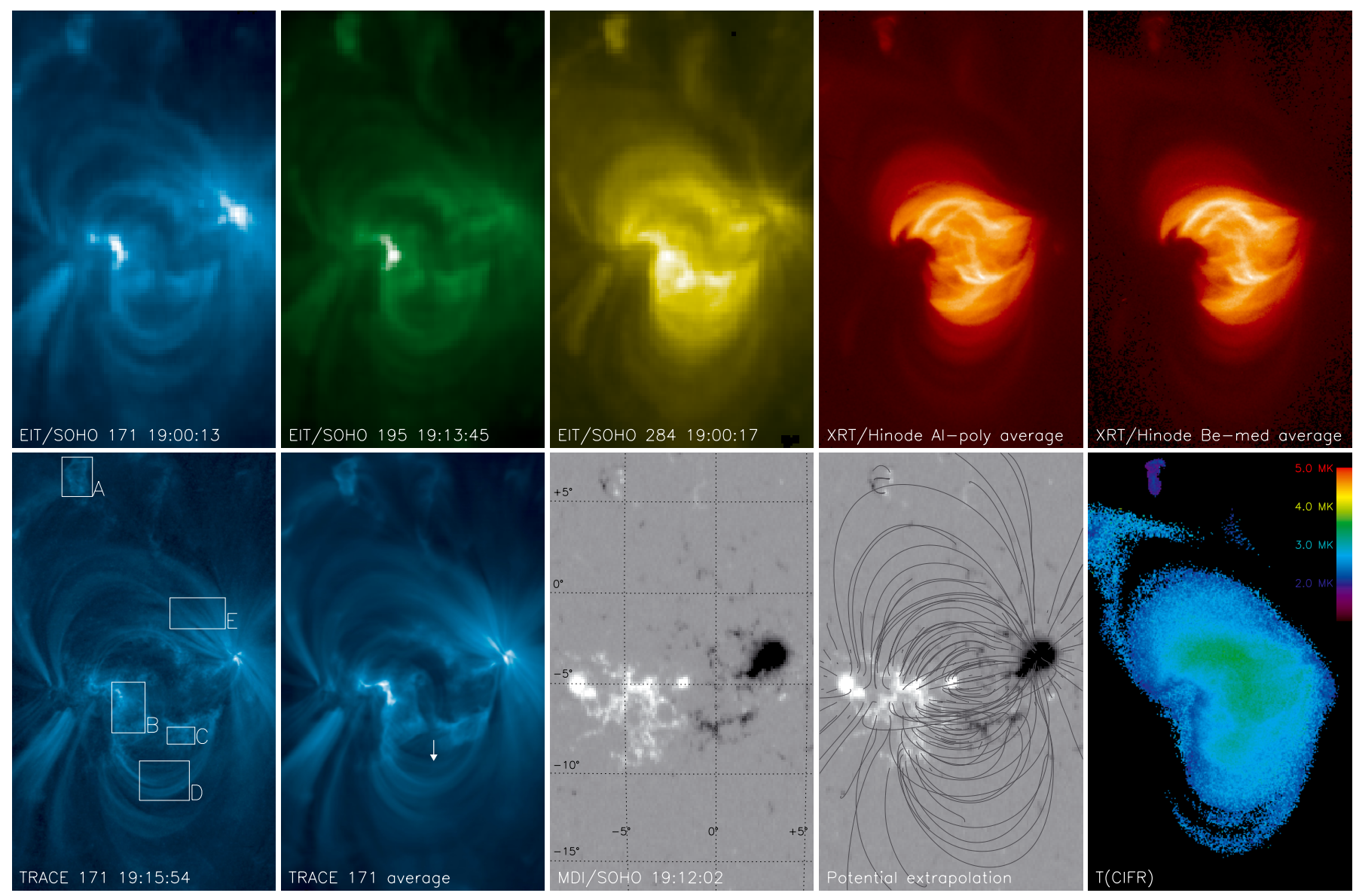

Fig. 1. Active region NOAA 10963. Top row, left to right: EIT/SOHO snapshots in EIT 171, EIT 195, and EIT 284 filters, XRT Al-poly and Be-med temporal average over the 18:00-20:00 UT period. Bottom row, left to right: snapshot in TRACE 171 filter at 19:15:54 UT, TRACE 171 temporal average over the period of 16:00 to 23:00 UT, MDI/SOHO magnetogram with the disk coordinate system, potential extrapolation of the magnetic field overlaid on the MDI/SOHO magnetogram, and the temperature structure of the active region obtained using the CIFR method. EIT, XRT, and TRACE observations are scaled with the square-root of intensity to enhance areas of weaker emission, e.g. the secondary bipole located near the upper right corner of individual images. TRACE 171 observations were deconvolved to remove the stray light. MDI/SOHO magnetogram is saturated to values of $\pm 1000 \mathrm{G}$. The arrow in the TRACE average image denotes loops that are discussed in Sect. 4.1.

To remove the effect of the Sun's rotation, all data were derotated to 19:00 UT, which corresponds to the time of observations in EIT 171 filter. For XRT data, the residual shifts due to the spacecraft jitter were removed using the xrt_jitter routine. We note that this routine gives almost identical results to the cross-correlation technique used by e.g. Warren et al. (2010).

The smoothing employed in the EIT data slightly reduced the resolution, which is $2.63^{\prime \prime}$ per EIT pixel. The resolution of XRT is about $1.02^{\prime \prime}$. The coalignment of the data from these two instruments was achieved manually for both the EIT 284 filter and XRT Be-thin filter. In order not to lose the accuracy of the coalignment, non-smoothed EIT 284 data were used. Thus, the maximum error in the coalignment is equal to 1 EIT pixel. The coalignment is insensitive to the choice of the XRT filter, since the observed distribution of emission is similar in all XRT filters. To illustrate this point, observations in the thinnest (Alpoly) and thickest (Be-med) XRT filters are displayed in Fig. 1.

The observed distribution of EUV and X-ray emission shown in Fig. 1 consists of a hot, bright X-ray active region core. The brightest structures within the core are non-potential loops connecting regions of opposite polarity. The core is surrounded on both sides by less bright loops. In the EIT 171, the core of the active region is relatively dim, except the bright moss regions located directly above plage polarities. Warm loops, both open and closed, are located on the peripheral parts of the active region. In
EIT 195 and EIT 284, the loops tend to concentrate progressively more in the active region core. The described morphology of active region emission is common for active regions (e.g., Warren \& Winebarger 2006, 2007; Ugarte-Urra et al. 2009; Brooks \& Warren 2009; Warren et al. 2010).

The secondary dipole is visible in all EIT and XRT filters, but the ratio of its maximum intensity to the maximum intensity observed in a given filter decreases with increasing temperature of the maximum of the filter response (Sect. 3.2). The ratio is about 0.40 for EIT 171, 0.34 for EIT 195, 0.21 for EIT 284, 0.10 for XRT Al-poly, and only 0.06 for the XRT Be-med filter.

The potential magnetic field extrapolation roughly captures the shape of warm coronal loops (Fig. 1), but fails to reproduce the shape of the brightest X-ray loops, which appear to be tangled. Thus, the distribution of the modeled emission in the active region core cannot be expected to closely reproduce the shape of these tangled loops. However, it can be expected to reproduce the spatial extent and intensity distribution of the active region core.

\subsubsection{TRACE 171 observations and the active region stability}

Modeling active region emission with steady models is meaningful only if the emission is stable within a given time interval. 

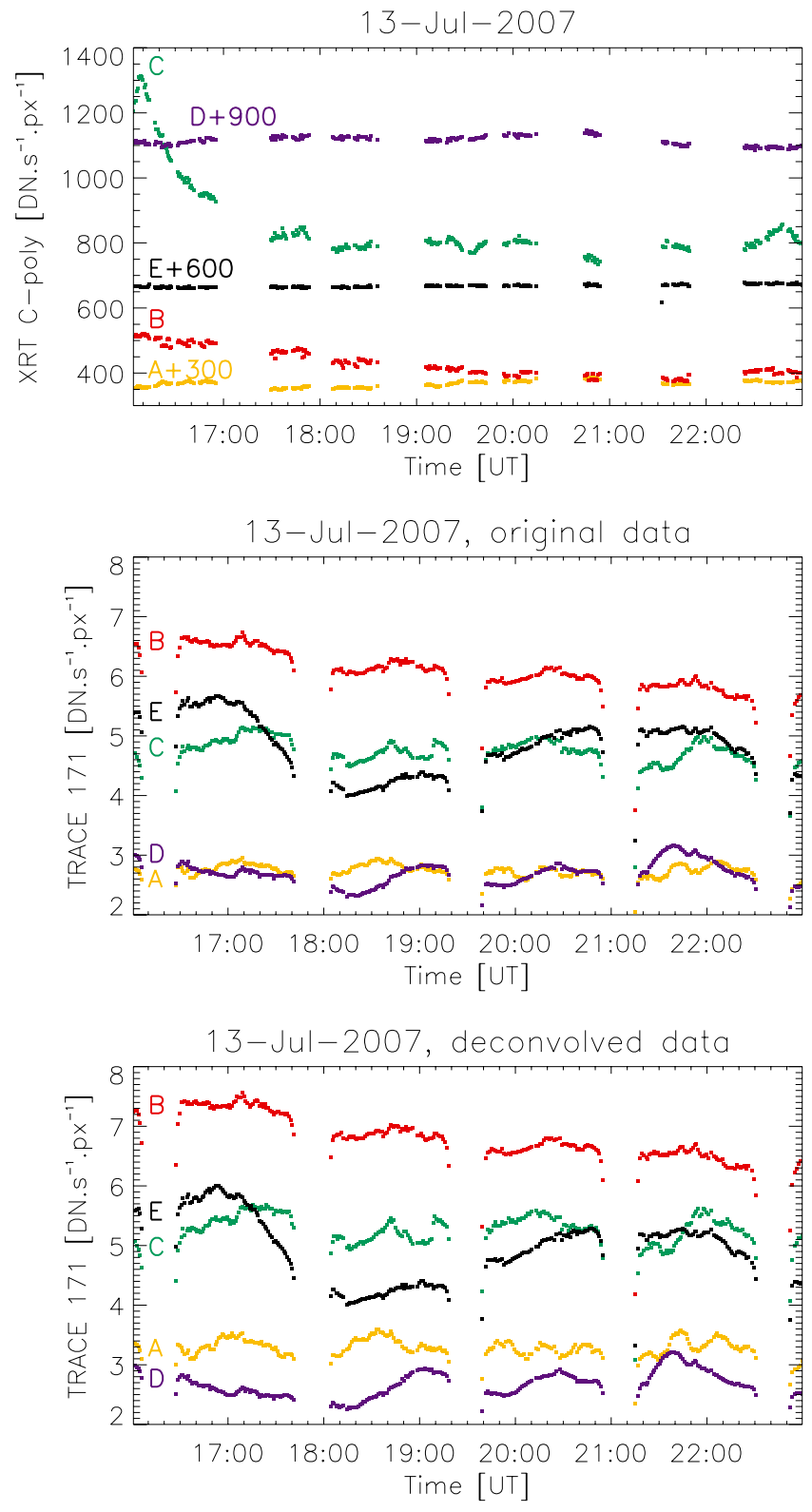

Fig. 2. Lightcurves for the five selected boxes, A-E (Fig. 1). Top: lightcurves for XRT C-poly data, middle: lightcurves for TRACE 171 data, bottom: lightcurves for TRACE 171 data with removed stray light. In the top image, the XRT C-poly lightcurves for boxes A, D, and E have been shifted by 300,600 , and $900 \mathrm{DN} \mathrm{s}^{-1} \mathrm{px}^{-1}$, respectively.

To determine whether the EIT "snapshots" (i.e., observations in only one time) can be used as an appropriate representation of active region emission, the evolution of the active region emission has to be studied. TRACE (Handy et al. 1999) observed the active region in the 171 filter with a cadence of approximately 1 min. There are on average about 40 observations per hour, with data gaps corresponding to the satellite night-time period. The observations were calibrated and normalized using the standard trace_prep routine, corrected for solar rotation, and deconvolved with the instrumental point-spread function of DeForest et al. (2009) to remove the stray light. The correction for rotation yields better results than cross-correlation with a single selected frame. The resolution of TRACE 171 data is $0.5^{\prime \prime}$, which is about 5.3 times better than the EIT resolution. Coalignment with EIT 171 is achieved using cross-correlation. An example of the TRACE 171 observation made at 19:15:54 UT is shown in Fig. 1. The peripheral loops, moss, and secondary bipole are clearly visible.

In principle, two direct checks of the variation in emission observed by a given filter can be performed. First one is the comparison of a given snapshot to the time-averaged image. The time-averaged TRACE 171 image is displayed in Fig. 1. The average image appears more "fuzzy" than a single snapshot. Although this indicates changes with time in both moss and coronal loop intensities, the overall emission morphology is similar. To quantify the magnitude of the emission variation, we selected five rectangular regions and constructed lightcurves of pixel-averaged intensities $\bar{I}$ over the time period of 16:0023:00 UT. In the construction of the lightcurves, we selected only pixels with signals greater than $1.5 \mathrm{DN} \mathrm{s}^{-1} \mathrm{px}^{-1}$ to remove background pixels. The lightcurves are the second possible way of checking the variation in the observed emission. The five selected rectangles encompass the secondary bipole (A), moss regions ( $\mathrm{B}$ and $\mathrm{C}$ ), and portions of the coronal loops ( $\mathrm{D}$ and E, Fig. 1). The area of the rectangles $A$ to $E$ is $507.3,711.5$, $197.7,830.1$, and $724.7 \mathrm{Mm}^{2}$, respectively. These areas are large enough for projection effects due to solar rotation to be neglected.

To study the effect of stray light, the lightcurves are constructed using the "standard" data with the stray light and data with the stray light removed. These lightcurves for TRACE 171 are displayed in Fig. 2 middle and bottom. It is obvious that the stray light affects the results. Its removal leads to changes in the relative intensity of various structures. The intensity of the secondary dipole increases on average by $19.9 \%$. The increase in intensity for the moss regions $\mathrm{B}$ and $\mathrm{C}$ is $12 \%$ and $11 \%$, respectively, while the regions $\mathrm{D}$ and $\mathrm{E}$ containing only coronal loops have an average intensity that is almost unchanged, being $-1 \%$ and $2 \%$, respectively, above the intensities for non-deconvolved data. Thus, in line with DeForest et al. (2009), we strongly recommend removing the stray light as a standard procedure for imaging instruments.

The variation in the selected rectangles A to E can be quantified by computing the percentage difference between the maximum and mean intensities, $\left(\bar{I}_{\max }-\langle\bar{I}\rangle\right) /\langle\bar{I}\rangle$, or the ratio of standard deviation to the mean intensity $\sigma(\bar{I}) /\langle\bar{I}\rangle$, where the upper bar and brackets denote the box-averaged and time-averaged values, respectively. The $\sigma(\bar{I}) /\langle\bar{I}\rangle$ quantity characterizes the dispersion in the lightcurves, while the $\left(\bar{I}_{\max }-\langle\bar{I}\rangle\right) /\langle\bar{I}\rangle$ is strongly biased towards brightest events (Ugarte-Urra et al. 2006). The values of these quantities for the five selected boxes are summarized in Table 3 for TRACE 171 data with removed stray light. For non-deconvolved data, the variability is generally smaller by a factor of $\sim 0.9$. We note that the values for $\mathrm{B}$ and $\mathrm{C}$ confirm the results of Antiochos et al. (2003), who found that the variations in moss intensities observed in TRACE 171 filter are only $\approx 10 \%$ over a period of five hours, and the results of Brooks \& Warren (2009), who found that the $\sigma(\bar{I}) /\langle\bar{I}\rangle$ for moss observed by Hinode/EIS (Culhane et al. 2007), using the line in line $195.12 \AA$, is less than $15 \%$ for individual macropixels over period of $16 \mathrm{~h}$.

The same analysis was performed using the data observed by XRT/Hinode in C-poly filter, except for the removal of stray light, since the point-spread function of the XRT telescope is not yet known. In this analysis, pixels with signal greater than $15 \mathrm{DN} \mathrm{s}^{-1} \mathrm{px}^{-1}$ are taken into account. The results are summarized in Table 3. The variability in boxes $\mathrm{A}, \mathrm{B}$, and $\mathrm{C}$ is greater than the variability seen in TRACE 171 . However, the $\sigma(\bar{I}) /\langle\bar{I}\rangle$ for boxes $\mathrm{B}$ and $\mathrm{C}$ is not greater than $\sim 15 \%$, similar to the values 
Table 3. Variability in selected boxes.

\begin{tabular}{lcccccc}
\hline \hline Filter & Ratio & A & B & C & D & E \\
\hline \multirow{2}{*}{ TRACE 171 } & $\frac{\sigma(\bar{I})}{\langle\bar{I}\rangle}$ & $4.7 \%$ & $6.0 \%$ & $5.3 \%$ & $8.3 \%$ & $11.4 \%$ \\
TRACE 171 & $\frac{\bar{I}_{\max }-\langle\bar{I}\rangle}{\langle\bar{I}\rangle}$ & $9.0 \%$ & $11.2 \%$ & $8.0 \%$ & $20.0 \%$ & $21.0 \%$ \\
\hline \multirow{2}{*}{ XRT C-poly } & $\frac{\sigma(\bar{I})}{\langle\bar{I}\rangle}$ & $13.9 \%$ & $11.3 \%$ & $15.3 \%$ & $6.3 \%$ & $7.5 \%$ \\
XRT C-poly & $\frac{\bar{I}_{\max }-\langle\bar{I}\rangle}{\langle\bar{I}\rangle}$ & $29.2 \%$ & $20.9 \%$ & $52.3 \%$ & $14.5 \%$ & $16.8 \%$ \\
\hline
\end{tabular}

found by previous authors for emission observed by XRT above the locations of moss in TRACE 171 (Brooks \& Warren 2009; Warren et al. 2010). The value $\left(\bar{I}_{\max }-\langle\bar{I}\rangle\right) /\langle\bar{I}\rangle=52.34 \%$ for the box $\mathrm{C}$ is caused by the greater emission around 16:10 UT. In the time interval 18:00-20:00 UT, the emission in box C is quite stable, as is the emission in other boxes.

We thus conclude that there are no significant changes in the emission of the active region around the time of the MDI/SOHO observations (Table 2), and for this reason, the active region is suitable for modeling under the assumption of steady heating.

\subsection{Filter responses to emission}

The emission observed by using EUV and X-ray filters can be linked to the plasma temperature $T$ and electron density $n_{\mathrm{e}}$ by the filter response to emission $F\left(T, n_{\mathrm{e}}\right)$ (e.g., Dudík et al. 2009b)

$F\left(T, n_{\mathrm{e}}\right)=\frac{1}{4 \pi} \iint g(\lambda) G\left(T, n_{\mathrm{e}}, \lambda, A\right) n_{\mathrm{e}}^{2} \mathrm{~d} \lambda \mathrm{d} l$,

where $\frac{1}{4 \pi}$ is a geometrical factor, $g(\lambda)$ is the combined spectral response of the telescope, filter and the detector, $\lambda$ denotes the wavelength, $l$ is the coordinate along the line-of-sight, and $G\left(T, n_{\mathrm{e}}, \lambda, A\right)$ is the spectral contribution function for a set of element abundances $A$, which we assume to be coronal. The value of $G\left(T, n_{\mathrm{e}}, \lambda, A\right) n_{\mathrm{e}}^{2}$ is the plasma emissivity per unit volume. The emissivity contains contributions from emission spectral lines and continuum, which is important mostly in the X-ray part of the spectrum. We use the CHIANTI atomic database and software, v5.2 (Dere et al. 1997; Landi et al. 2006) to calculate the contribution function. The response functions for EIT and XRT filters used in this paper are shown in Fig. 3 top and middle for the assumed value of emission measure $10^{27} \mathrm{~cm}^{-5}$. The XRT responses are functions of $T$ only, i.e., they are relatively independent of $n_{\mathrm{e}}$, which comes from the behavior of X-ray spectrum. The possible influence of various particle distributions (e.g., $k$ distributions) on the filter responses (Dudík et al. 2009b) is not considered in this paper.

The XRT responses are time-varying because of the dependence on the CCD contamination layer, which changes the instrument's spectral response $g(\lambda)$. This is taken into account during the construction of the XRT responses using the standard make_xrt_wave_resp routine of the SolarSoft. The contamination layer on the CCD of the EIT telescope is not taken into account, since this option is not provided by the eit_parms routine.

If the distributions of temperature $T(l)$ and density $n_{\mathrm{e}}(l)$ along the line of sight are known, Eq. (19) can be used to calculate the observed signal in the given filter. In general, the inverse problem is not easily solved for coronal plasma, since the observed signal is an integral along the line of sight (but see e.g. Vásquez et al. 2009; Aschwanden et al. 2009).
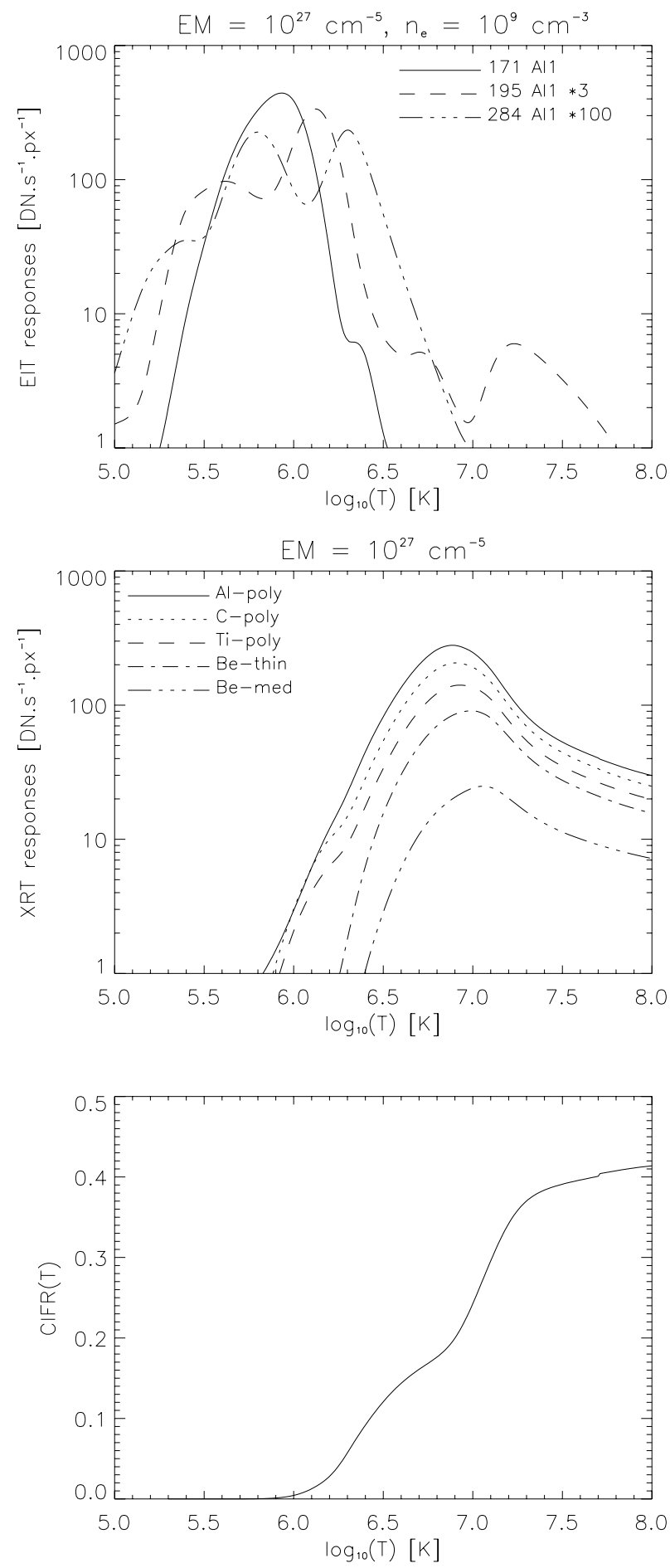

Fig. 3. Top: responses of the EIT filters to $T$ for $n_{\mathrm{e}}=10^{9} \mathrm{~cm}^{-3}$. Middle: responses of five XRT filters to $T$. The XRT filter responses are independent of $n_{\mathrm{e}}$. Both EIT and XRT responses are normalized to the emission measure of $10^{27} \mathrm{~cm}^{-5}$. Bottom: combined improved filter ratio constructed from the five XRT filter responses.

In this work, we use Eq. (19) to convert the obtained temperature and density distributions (Sect. 2.2) to the predicted signal. To this end, we computed the wavelength-integrated quantity $F^{\prime}\left(T, n_{\mathrm{e}}\right)=n_{\mathrm{e}}^{2} \int g(\lambda) G\left(T, n_{\mathrm{e}}, \lambda, A\right) \mathrm{d} \lambda$ for a logarithmically distributed values of $T$ and $n_{\mathrm{e}}$. The distribution of temperatures and densities, $T(x, y, z)$ and $n_{\mathrm{e}}(x, y, z)$ obtained in the manner described in Sect. 2 are then converted to the distribution of 
$F^{\prime}(x, y, z)$. The distribution of $F^{\prime}(x, y, z)$ is then multiplied by the voxel volume and integrated along the line of sight $l \equiv z$ to obtain the distribution $F(x, y)$ of the predicted signal. We note that in the computation of $F^{\prime}(x, y, z)$, no additional correction for expanding loop geometry is neccessary, because a given field line is used to compute $T(x, y, z)$ and $n_{\mathrm{e}}(x, y, z)$ only at one point corresponding to a given voxel $(x, y, z)$ and the voxels are uniformly spaced.

\subsection{Temperature diagnostic using CIFR}

In principle, the temperature can be diagnosed from observations in multiple filters, if the ratio of filter responses is a monotonic function of temperature. This is not in general true for EUV filters, but possible for X-ray filters. To optimize the signal-tonoise (hereafter $\mathrm{S} / \mathrm{N}$ ) ratio, Reale et al. (2007) proposed the combined improved filter ratio (CIFR) method, which evaluates the square of the geometric mean of five filter responses divided by the responses of the two thinnest filters

$\operatorname{CIFR}(T)=\frac{\left(\prod_{n=1}^{5} F_{i}(T)\right)^{2 / n}}{F_{1}(T) F_{2}(T)}$.

In this paper, $F_{1}$ is Al-poly, $F_{2} \mathrm{C}$-poly, $F_{3} \mathrm{Ti}$-poly, $F_{4} \mathrm{Be}$-thin, and $F_{5}$ Be-med filter responses. The $\operatorname{CIFR}(T)$ curve is independent of $n_{\mathrm{e}}$ and is for these five filters depicted in Fig. 3 bottom. Under the assumption that the plasma is isothermal, the temperature map of the active region can be constructed simply by computing the value of CIFR for each pixel and finding the corresponding $T$ (e.g., Parenti et al. 2010). The temperature map of the active region 10963 is depicted in Fig. 1.

In practice, this method will not detect temperatures below the value of $\log _{10}(T) \approx 6.2$ because of to filter response limitations. This lower limit is given by the response of the XRT Bemed filter, which is the thickest one used here (Fig. 3 middle). The actual value of the lower temperature limit is to some extent set by the highest acceptable $\mathrm{S} / \mathrm{N}$ ratio in each filter, which we take to be equal to $(1 / 10)^{1 / 2} \approx 31.6 \%$. The temperature structure in Fig. 1 is plotted only for pixels where the relative error in the XRT signal is in all filters lower than this value. This is done to exclude the areas of weak signal with very low $\mathrm{S} / \mathrm{N}$ ratio.

The assumption that the coronal plasma is isothermal is almost never satisfied, since it is optically thin. In the least, contamination from diffuse background and/or foreground emission is present, or the plasma is multithermal (e.g., Schmelz et al. 2005; Schmelz \& Martens 2006; Cirtain et al. 2007; Schmelz et al. 2007a, 2008; Aschwanden et al. 2008; Schmelz et al. 2009a; Terzo \& Reale 2010; O’Dwyer et al. 2010), or there is a contribution from low-intensity high-temperature component (e.g. Reale et al. 2009a,b; Schmelz et al. 2009b,c). The effects of plasma multithermality are discussed in Reale et al. (2007) and for TRACE in e.g. Weber et al. (2005) and Schmelz et al. (2007b). We return to the assumption of isothermal plasma in Sect. 4.4, where the performance of the CIFR method is evaluated.

\section{Results}

Using the approach outlined in Sect. 2, we constructed an array of models spanning wide ranges of free parameters: $-4.0 \leq \log _{10}\left(C_{\mathrm{H} 0}\right) \leq-2.0$, with the step of $\Delta \log _{10}\left(C_{\mathrm{H} 0}\right)=0.25$, $0.25 \leq \rho \leq 2.0$, with the step of $\Delta \rho=0.25$, and $0 \leq \tau \leq 2$, with the step $\Delta \tau=0.25$. In addition, for $0.5 \leq \rho \leq 1.0$, the models are computed with $\Delta \rho=0.1$. Altogether, this translates into 972 models.
For each of these models, we computed the synthetic EIT and XRT emission in three and five filters, respectively, and derived the synthetic $T(C I F R)$ map from the synthetic XRT filtergrams.

The computational box has $121 \times 201 \times 91$ uniformly spaced voxels. The horizontal size of the computational box corresponds to the portion of the MDI/SOHO, EIT/SOHO, TRACE, and XRT/Hinode observations shown in Fig. 1. Resolution of the computational box corresponds to the resolution of the MDI/SOHO instrument, $1.43 \mathrm{Mm} \mathrm{px}^{-1}$.

\subsection{Best-fit to the observed emission}

Finding a single model consistent with all observations is a challenging task because of the vast number of produced images. Our strong selective criterion was that of a suitable temperature structure. We found that if the $T(C I F R)$ derived from synthetic $\mathrm{X}$-ray filtergrams does not correspond to the $T(C I F R)$ derived from the observations, then the given model does not reproduce all or any of the observations. This emphasizes the primary role of the temperature structure of the active region in constraining the coronal heating problem.

After retrieving only the models with the appropriate temperature structure, a best-fit model is found by a visual comparison with the observations. We note that Warren \& Winebarger (2006) utilized the dependence of the total modeled X-ray intensity on the unsigned photospheric magnetic flux for 26 active regions as a criterion for selection among various parametrisations of the heating rate. However, these authors found that visualizations and their comparisons to observations are neccessary to select among models with total intensity matching the total observed intensity. We are thus motivated to use the visualizations of the predicted emission distribution relative to observations as a strong selection criterion.

The suitable models must satisfy our following conditions for visual comparison:

1. the spatial extent of the X-ray core must be neither too small nor too large compared to the observations. The brightest, tangled loops in the center are not expected to be reproduced accurately because of the potential extrapolation (Sects. 2.1 and 3.1.1);

2. strong X-ray emission should not appear outside of the core of the active region;

3. all three components of the EUV emission, i.e., coronal loops, moss, and the secondary dipole must be present;

4. the intensity of the moss and the secondary dipole should be neither too bright nor too faint compared to each other, to the coronal loops, and also to the observations.

We emphasize that our visual criteria concern only the global properties of the active region emission, since a detailed match is not possible owing to the potential approximation. We also remind the reader again that the emission models do not contain open loops, which are discarded (Sect. 2.3), and that the visual agreement is sought only for models with a suitable temperature structure.

In practice, several tens of models yield appropriate temperature structure. These models are clustered within a small parameter range. Applying the listed criteria for visual comparison reduces this parameter range to only a few models with $\rho=0.6-0.8$ and $\tau=0.25-0.5$. We find that the closest visual fit is achieved for $\rho=0.7-0.8, \log _{10}\left(C_{\mathrm{H} 0}\right)=-3.0$, and $\tau=0.5$. The comparison with observations is displayed in Fig. 4 and the comparison of the modeled and observed temperature structure derived using CIFR method is displayed in the first column of 

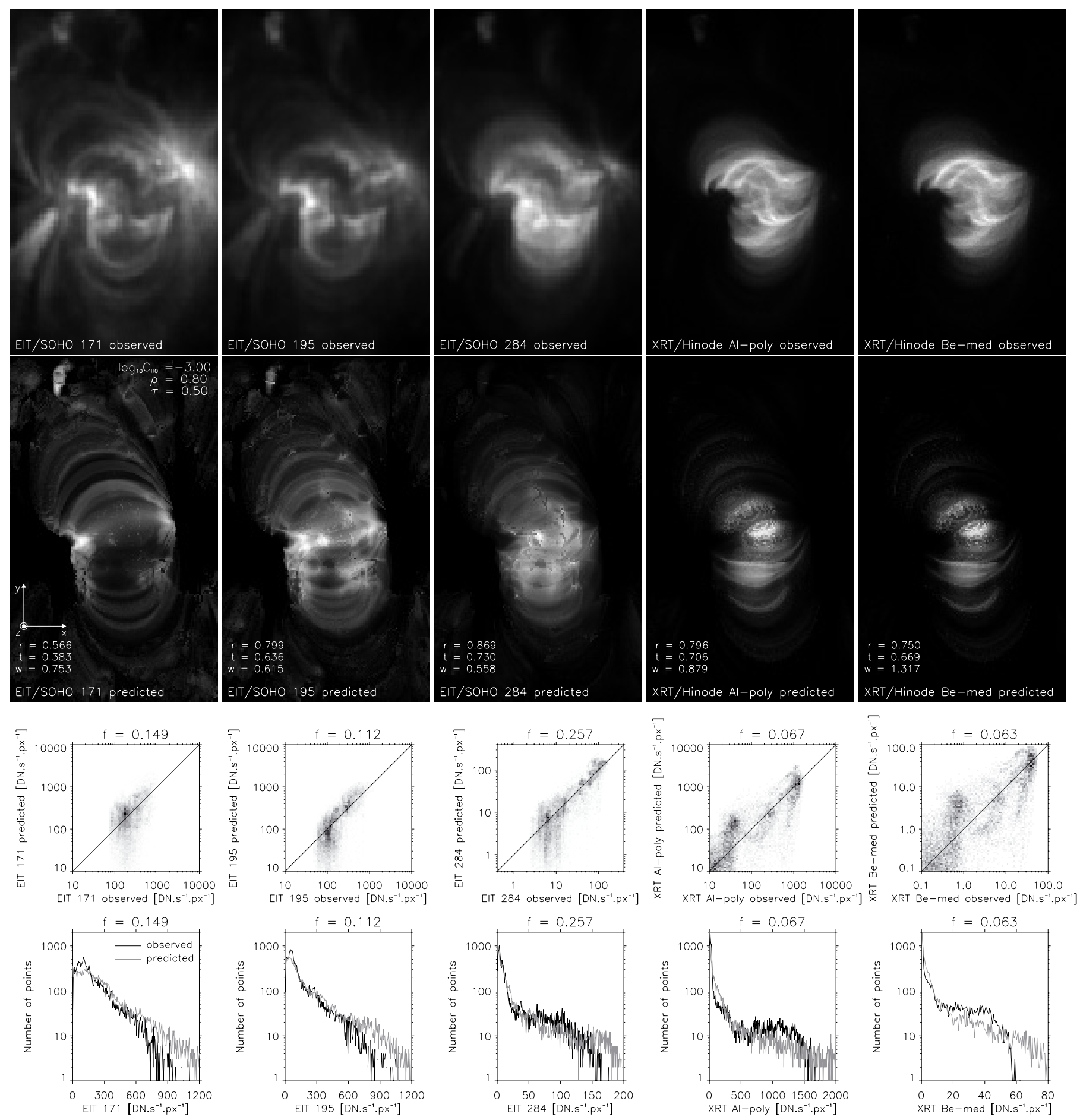

Fig. 4. Best-fit to the observations. Top row: observations in five filters shown in linear intensity scale. Second row: predicted (synthetic) intensity images. Third row: probability density function of the predicted intensity as function of the observed intensity. Black color represents higher concentration of points. Bottom row: histograms of the observed (black line) and predicted intensity distributions (gray line). The filling factor $f$ used to scale the predicted intensity is listed in individual graphs.

Fig. 5. Temperature structure is very sensitive to any change in these parameters, even if the changes in the resulting emission are small. The influence of various free parameters on the modeled temperature structure and the synthetic emission is discussed in Sect. 4.2.

The predicted (synthetic) emission is quite high compared to the observed signal. This is a well-known property of both the static and dynamic models and is usually attributed to the presence of a filling factor $f$ (e.g., Porter \& Klimchuk 1995; Warren \& Winebarger 2006, 2007; Warren et al. 2008; Lundquist et al. 2008a,b; Winebarger et al. 2008; Warren et al. 2010). The filling factor can also be determined from observations (e.g., Tripathi et al. 2009). We determine the filling factor as the ratio of the mean observed intensity to the mean predicted intensity. The filling factors for each filter are different, being smallest for X-ray filters ( 0.067 for Al-poly and 0.063 for Be-med filter), and 
J. Dudík et al.: Is it possible to model observed active region coronal emission simultaneously in EUV and X-ray filters?
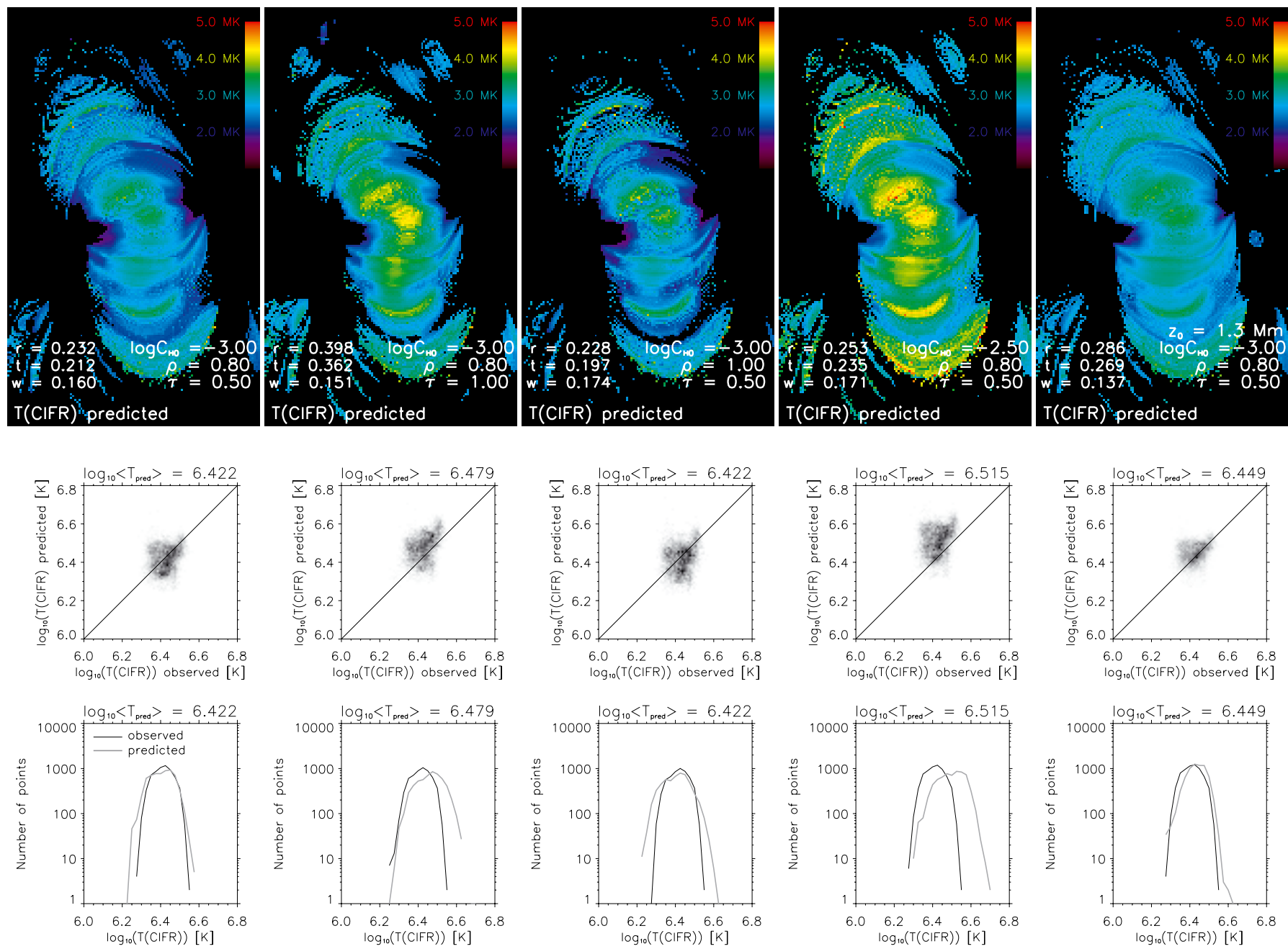

Fig. 5. Temperature distributions derived from five various models using the CIFR method. Each column corresponds to a different model. The model parameters are listed in the images at the top. Top row: temperature distribution maps. Middle row: probability density function of the temperature distributions derived from the predicted and observed X-ray emission. Bottom row: temperature distribution histograms.

slightly larger for EIT filters (0.149 for EIT 171, 0.112 for EIT 195 , and 0.257 for EIT 284). We note here that some of the discrepancy in the filling factors may be caused by errors in the EIT calibration (Sect. 2.1), with the EIT 284 data being most affected by the contamination. We also note that according to the observations, a plasma with $T(C I F R) \approx 2-3 \mathrm{MK}$ occupies most of the area on the temperature map (Fig. 1). The increase in $f$ in the 1-2 MK interval is also consistent with the results of Schrijver et al. (1999) based on inspection of TRACE observations. The existence of the filling factor in our model means that only a portion of the voxel volume is filled with heated plasma. The filling factor can account for both the possibility of substructuring, i.e., multi-strand loops with wide-ranging densities in different strands, or for the possibility that only a small portion of the active region coronal volume is heated at a given time (Warren \& Winebarger 2006). However, without sufficiently high magnetogram resolution, we cannot determine which situation corresponds to reality. The $n_{\mathrm{e}}^{2} f$ and possibly $T$ obtained by our model in a given voxel should then be taken only as average quantities for this voxel.

Following Lundquist et al. (2008a,b), the level of agreement between the model and the observations can be quantified by the correlation coefficient $r$, rank correlation coefficient $t$, and the weighted-rms relative error $w$. We modify the computation of $w$

$w=\sqrt{\frac{\sum_{i}\left(I_{\mathrm{obs}, i}-f I_{\mathrm{pred}, i}\right)^{2} / I_{\mathrm{obs}, i}}{\sum_{i} I_{\mathrm{obs}, i}}}$

by the inclusion of the filling factor $f$. The correlation coefficients $r$ and $t$ are independent of $f$. To compute these values, the observations are regridded to match the resolution of the computational box. The obtained values of $r, t$, and $w$ are listed in each synthetic image in Figs. 4-7.

We note that the values obtained for our best-fit model, namely $r \approx 0.8$ and $w \lesssim 1$ are better than those obtained by Lundquist et al. (2008a) and listed in their Table 4. However, they consider a different heating function and also a different instrument (SXT/Yohkoh). For heating scaling as $B_{0} / L_{0}$, we obtain similarly "worse" values of $w \approx 1-1.5$ as Lundquist et al. (2008a) do, but our synthetic images do not correspond to the observations for such a scaling.

Another way of quantifying the level of agreement between the model and observations is to perform a pixel-to-pixel comparison of intensities and intensity histograms as shown in Fig. 4. 

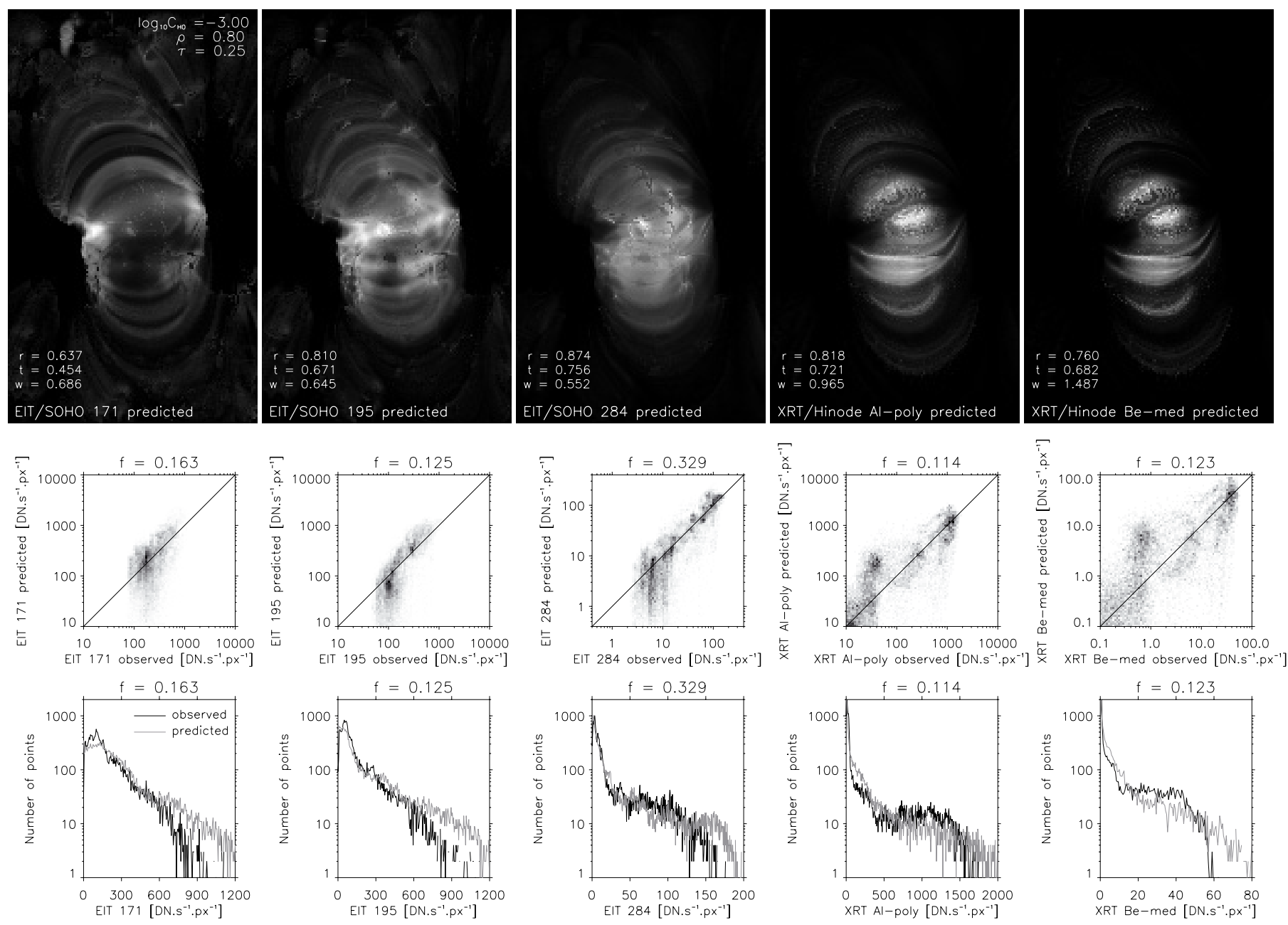

Fig. 6. Similar as in Fig. 4, but for $\tau=0.25$.

The pixel-to-pixel comparison is plotted in the form of a probability density function (pdf), i.e., the density of points on the scatterplot. The pdf allows us to discern a higher concentration of points (darker color) rather than display a simple scatterplot as a cloud of points. The probability density function follows the $I_{\text {pred }}=I_{\mathrm{obs}}$ line on the plots only roughly. The situation is at its worst for the X-ray filters, where the potential approximation breaks down for the tangled loops. The intensity histograms for all filters are much more accurately reproduced.

The best-fit model captures the following properties of the observed active region emission: (1) the general emission morphology, with a hot X-ray active region core that is very similar in the Al-poly and Be-med filters; (2) the existence of warm, closed coronal loops located at the northern and southern periphery of the active region core, with the longest loops seen in the 171 filter being cooler and lying more on the periphery than shorter, warmer loops in EIT 195 and 284; (3) unstructured intensity profile and constant cross-section of coronal loops; (4) existence of moss, which is brightest in the Fe XII and Fe XIII lines (Tripathi et al. 2010), i.e., in EIT 195; and (5) progressive lowering of the intensity of the secondary bipole with increasing maximum temperature of the filter response.

Apart from the detailed comparison, the modeled emission differs from observations in several aspects. These include (1) the presence of warm, diffuse loop-like emission overlying the $\mathrm{X}$-ray active region core; (2) the presence of warm loops mainly in EIT 171 in areas where none are observed; and (3) discrepancies in the intensities of the secondary bipole and moss emission.

The overlying diffuse, loop-like emission comes from a diffuse bundle of $\approx 0.9-1.4 \mathrm{MK}$ loops located at heights of 30 $60 \mathrm{Mm}$ above the chromosphere. Loops in this temperature range are known to exist in active regions, being a distinct population from the core X-ray loops (Ugarte-Urra et al. 2009). However, such loops are not prominent in observations of our active region, although some loops are observed in this place in the TRACE 171 seven-hour average image (Fig. 1). The presence of such loops can be a consequence of the potential extrapolation, which is not valid in locations where tangled X-ray loops are observed. This is because a different field geometry leads to changes in the values of loop half-lenghts $L_{0}$ and also to changes in the properties of the field, i.e., the rate of field decrease $s_{\mathrm{H}}$ and expansion $\Gamma$, which are in our model directly linked to the predicted emission through the temperature and density distributions (Sect. 2).

The loops predicted in the vicinity of the box D in Fig. 1 are not observed by EIT 171. However, similar loops are visible in the TRACE 171 seven-hour average image. These loops lie $\approx 5^{\prime \prime}$ southwards of the predicted ones and are indicated with an arrow in Fig. 1. The emission of these loops peaks at approximately 17:15 and 22:00 UT. This could indicate that not all predicted warm loops are visible at a given time.

The secondary bipole is very bright in the predicted EIT 171 and 195 emission, and has no intensity predicted in the XRT 
J. Dudík et al.: Is it possible to model observed active region coronal emission simultaneously in EUV and X-ray filters?

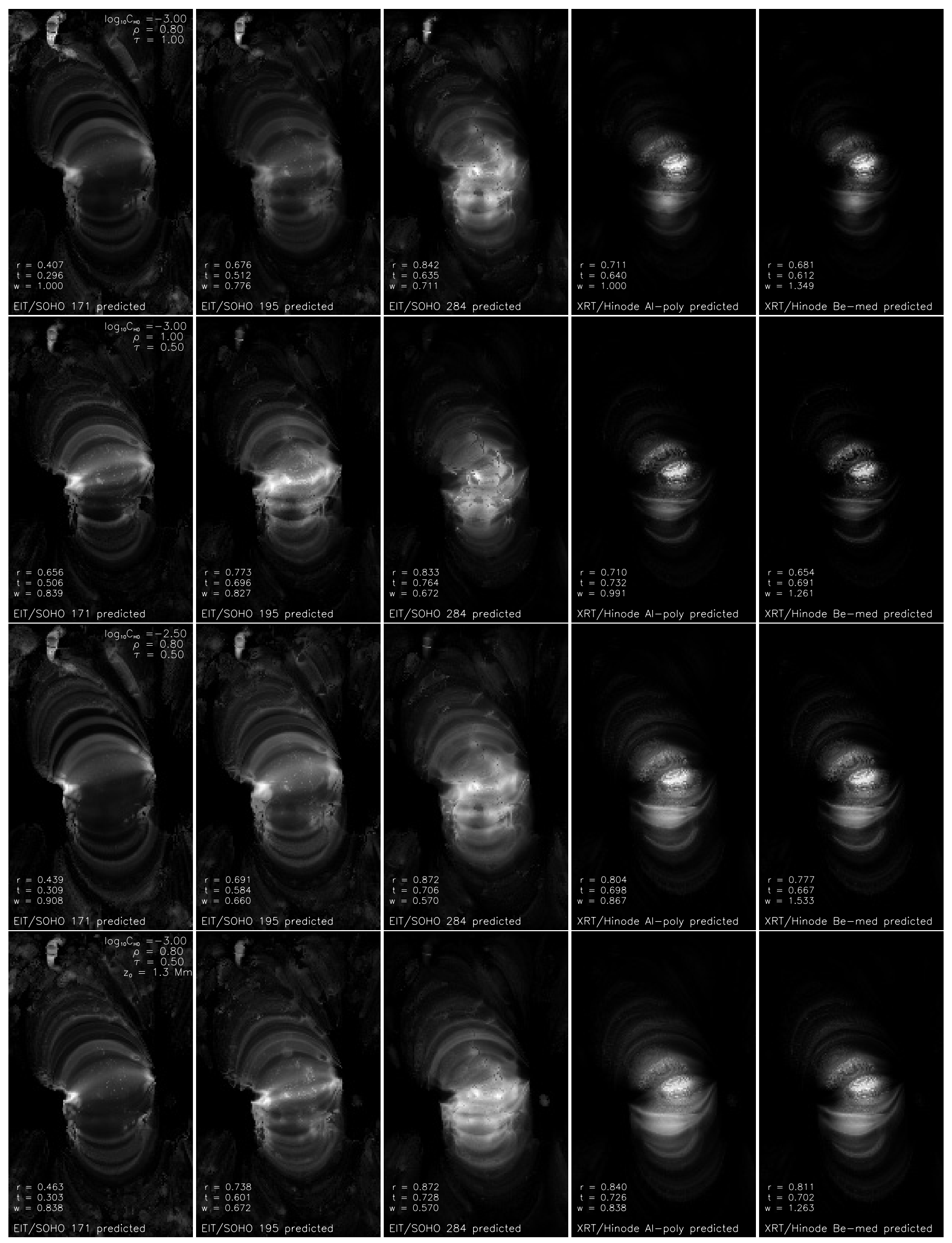

Fig. 7. Role of the heating parameters. Top row: $\tau=1$, second row: $\rho=1$, third row: $\log _{10}\left(C_{\mathrm{H} 0}\right)=-2.5$, bottom row: chromosphere lowered to $z_{0}=$ $1.3 \mathrm{Mm}$. The parameters of the heating function in Eq. (13) are listed in each row. 
Be-med filter. The ratio of its maximum intensity to the maximum intensity predicted for the entire active region is 0.86 for EIT 171, 0.70 for EIT 195, 0.16 for EIT 284, 0.02 for XRT Alpoly, and 0 for XRT Be-med filter. In general, both the secondary dipole and moss intensities are very sensitive to the value of $\tau$, but in the opposite sense: if $\tau$ decreases, the dipole intensity decreases, but the moss intensity increases. Lowering the value of $\tau$ to 0.25 would lower the dipole intensity ratio to be closer to the observed values for EIT 171 and 195, and increase the moss intensity in EIT 171, but would also lead to an increase in the intensity of the peripheral X-ray loops (Fig. 6), outside the observed spatial distribution of the X-ray emission, violating the second criterion for visual comparison.

\subsection{Role of the free parameters $\rho, \tau, C_{\mathrm{H} 0}$, and $z_{0}$}

To demonstrate the influence of the free parameters of the heating function represented by Eq. (13) on the resulting emission and temperature distributions, we change each of these parameters while keeping the other two the same as in the best-fit model. For $z_{0}=3 \mathrm{Mm}$ (Sect. 3.2), we chose models with $\tau=1.0(\rho=0.8$, $\left.\log _{10}\left(C_{\mathrm{H} 0}\right)=-3.0\right)$, with $\rho=1\left(\tau=0.5, \log _{10}\left(C_{\mathrm{H} 0}\right)=-3.0\right)$, and with $\log _{10}\left(C_{\mathrm{H} 0}\right)=-2.5(\rho=0.8, \tau=0.5)$. The emission distributions in five filters are displayed in Fig. 7 and the corresponding temperature structure in Fig. 5.

The temperature structure is displayed only for points where the relative error in the predicted signal in all of the XRT filters is less or equal to $(1 / 10)^{1 / 2} \approx 31.6 \%$. This is neccessary to avoid errors due to low signals with high photon noise. The histograms of the observed and modeled temperature structure are displayed in Fig. 5 bottom row only for points where the relative error is greater than this value in both the observations and the model prediction. Since the spatial distribution of the predicted signal varies with the parameters of the heating function, the number of pixels where the predicted signal is high enough is different for each model. This causes slight variations in the histogram of the observed temperature structure.

Increasing $\tau$ to 1 further enhances the emission of the secondary bipole, but suppresses the intensity of the moss. The temperature of shorter, core loops increases from approximately $4 \mathrm{MK}$ to $5 \mathrm{MK}$. The temperature distribution moves to higher temperatures (Fig. 5 second column).

Increasing $\rho$ to 1 steepens the dependence of the emission on the magnetic field in the footpoints, $B_{0}$, and shortens the scale-lengths $s_{\mathrm{H}}^{\Gamma}$. As a result, the temperature distribution widens (Fig. 5 third column). The emission distribution slightly contracts spatially, i.e. shorter loops become apparent. The EIT 171 intensity of the diffuse loop-like structure overlying the active region core increases, making it even more prominent.

Increasing the base heating rate to $\log _{10}\left(C_{\mathrm{H} 0}\right)=-2.5$ moves the temperature distribution to higher temperatures (Fig. 5 fourth column), since $T_{1} \sim\left(C_{\mathrm{H} 0}\right)^{2 / 7}$ to a first approximation (Eqs. (9) and (13)). As a result, the emission morphology changes, different loops can become visible, and the moss intensity in EIT 171 and 195 decreases significantly.

The height of the chromosphere-transition region boundary, $z_{0}$, can also be considered a free parameter of the model. In essence, it controls how many horizontal layers in the computational box near the photosphere will be discarded. For $z_{0}=3 \mathrm{Mm}$ (Sect. 3.2), the discarded voxels are the ones with $z=0$ and $z=1$. To study the effect of $z_{0}$, we compute a model with $z_{0}=1.3 \mathrm{Mm}$ (Aschwanden \& Schrijver 2002), which retains voxels with $z=1$. Since decreasing the $z_{0}$ increases $L_{0}$, the temperature distribution moves slightly to higher temperatures (Fig. 5 last column). It also decreases the intensity of the moss and increases the emitting volume of the secondary bipole, which becomes a prominent emitting feature in the EIT 171 synthetic image (Fig. 7 last row). We note that the height of the transition region in the model of Gudiksen \& Nordlund (2005a) varies between 2.7-12.3 Mm, with an average of $5 \mathrm{Mm}$. In our case, the predicted emission after adopting $z_{0}=5 \mathrm{Mm}$ would be smoother because of the absence of additional layers, but it would also again lead to the disappearance of the moss. We thus consider $z_{0}=3 \mathrm{Mm}$ to be an adequate value for the modeling of the active region emission and note that the transition region appears only at $z_{0}>2 \mathrm{Mm}$ in the standard chromospheric models (e.g., Vernazza et al. 1981; Avrett \& Loeser 2008).

\subsection{Role of the $s_{\mathrm{H}}$ in the structure of the active region corona}

It is known that uniform heating cannot reproduce the observed EUV emission of the active region (e.g., Warren \& Winebarger 2006). In the resulting emission models, the predicted intensity in the EUV filters are dominated by moss, and there are almost no coronal loops present. In our model, fixing the scale-length $s_{\mathrm{H}}^{\Gamma}$ or the ratio $L_{0} / s_{\mathrm{H}}^{\Gamma}$ to a pre-defined value would lead to diffuse emission simply because $T_{1} \sim B_{0}^{2 \rho / 7}$ and $p_{0} \sim B_{0}^{6 \rho / 7}$, according to Eqs. (9), (10), and (13). Computing the $s_{\mathrm{H}}$ from the magnetic field decrease along the given field line ties the $s_{\mathrm{H}}$ to the magnetic structure of the active region corona. Using the $s_{\mathrm{H}}$ computed in this way along with the loop expansion factor $\Gamma$ allows us to approximate the structure of the magnetic field during computation of the distributions of $T$ and $n_{\mathrm{e}}$. Since the magnetic field is spatially variable, $s_{\mathrm{H}}$ and $\Gamma$ are variable as well. The structure of temperature and density distributions $T(x, y, z)$ and $n_{\mathrm{e}}(x, y, z)$ within the computational box then strongly depend on the spatial structure of the $s_{\mathrm{H}}^{\Gamma}(x, y, z)$.

The dependence of the EIT 171 and XRT Al-poly signal in individual voxels on the value of $L_{0} / s_{\mathrm{H}}^{\Gamma}$ is plotted in Fig. 8 left and middle. This figure shows that the EIT 171 signal originates dominantly from voxels where $L_{0} / s_{\mathrm{H}}^{\Gamma} \gtrsim 5$, while the strongest XRT Al-poly signal is produced by loops for which $L_{0} / s_{\mathrm{H}}^{\Gamma} \lesssim 5$. The reason for this is easy to understand: according to the scaling law for $T_{1}$ (Eq. (9)), higher values of $L_{0} / s_{\mathrm{H}}^{\Gamma}$ lead to lower values of $T_{1}$, because $\gamma_{2}>0$. There are several voxels where the EIT 171 signal is strong for $L_{0} / s_{\mathrm{H}} \lesssim 5$ because of the high density in such voxels, as can be inferred from comparison of Fig. 8 left and right.

That EIT 171 and XRT signals are formed predominantly in loops with different $L_{0} / s_{\mathrm{H}}^{\Gamma}$ shows that the spatial variability of $s_{\mathrm{H}}^{\Gamma}$ in this work is important for capturing the emission properties of the active region. It could also explain why the 171 and X-ray observations are often "complementary" (e.g., Reale et al. 2007; Martens 2010), i.e., the signals in 171 and X-ray filters are observed at different locations. According to Fig. 8 right, this is because for a given $n_{\mathrm{e}}$, the signal in EIT 171 and XRT Al-poly is anticorrelated because of their dependence on $T$ and thus on the $L_{0} / s_{\mathrm{H}}^{\Gamma}$ ratio.

Applying the stability criterion in Eq. (17) for coronal loops, we found that all voxels with $L_{0} / s_{\mathrm{H}}^{\Gamma} \gtrsim 10$ correspond to unstable loops that should undergo the condensation-evaporation cycle. According to Fig. 8 left, the majority of the loops seen in EIT 171 fall into the unstable category, while according to Fig. 8 middle, virtually the entire XRT Al-poly signal originates from stable loops. In addition, while a large part of the 195 loops correspond to unstablesolutions (not shown), 

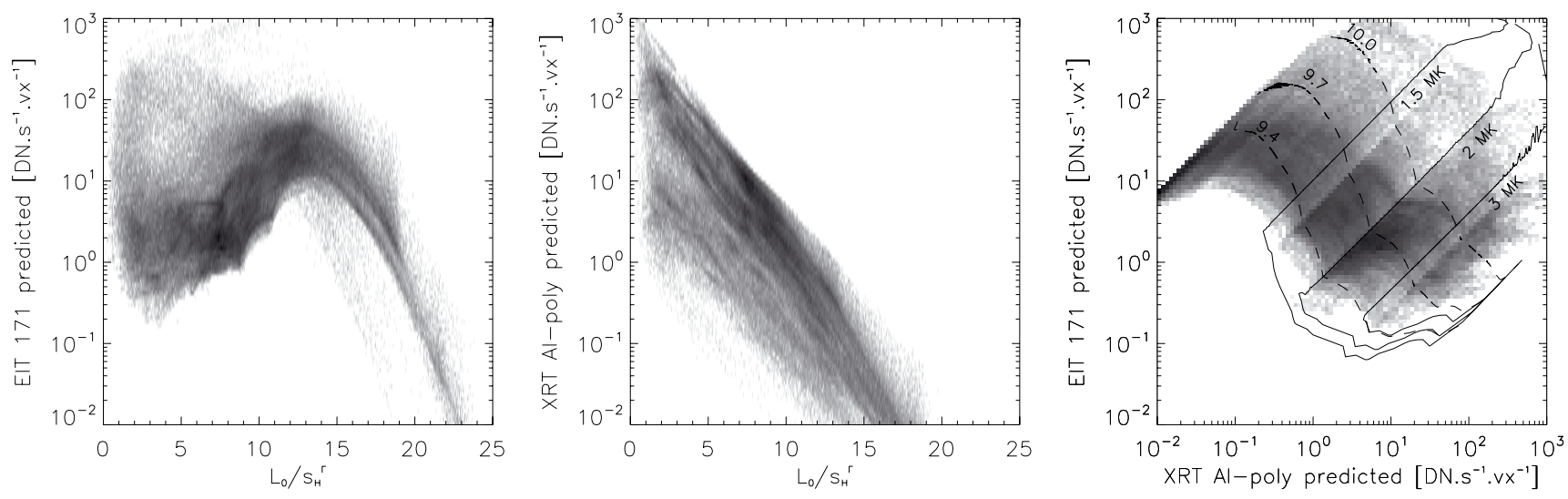

Fig. 8. Predicted signal in EIT 171 and XRT Al-poly filters in individual voxels. Left: logarithm of the probability density function of the dependence of the EIT 171 predicted signal on $L_{0} / s_{\mathrm{H}}^{\Gamma}$. Middle: same as left, but for the XRT Al-poly filter. Right: logarithm of the probability density function of the interdependence of the EIT 171 and XRT Al-poly signal in individual voxels. Density contours are shown as dashed lines (labels are in $\mathrm{cm}^{-3}$ ), temperature contours are shown as full lines.

the majority of the 284 loops are stable. These results could explain:

1. why Aschwanden et al. (2001) and Winebarger et al. (2003) found that only minority (approximately 30\%) of the 171 and 195 loops are consistent with steady-state solutions of Eq. (2);

2. why the models based on the steady-state hydrodynamic solutions of Eq. (2) are successful in reproducing the X-ray emission morphology (e.g., Warren \& Winebarger 2006, 2007; Lundquist et al. 2008a,b);

3. why the hot, X-ray active region core and the associated moss appear to be heated steadily (e.g., Antiochos et al. 2003; Warren et al. 2010); and

4. why the X-ray emission is usually not located above the sunspot umbra, where very short $s_{\mathrm{H}}^{\Gamma}$ are found in our model.

However, the thermally unstable solutions are problematic. We discuss them, together with their important implications, in Sect. 5.3 .

\subsection{CIFR performance evaluation}

The model of $T(x, y, z)$ and $n_{\mathrm{e}}(x, y, z)$ and the corresponding emission enables us to evaluate the performance of the $C I F R(T)$ temperature diagnostic method of Reale et al. (2007, Sect. 3.3).

To do this, we calculated the following quantities for each image pixel $(x, y)$ of the best-fit model (Sect. 4.1): the average temperature $T_{\mathrm{avg}}(x, y)$ along the line of sight $z$, the maximum temperature $T_{\max }(x, y)$, the emission-measure-weighted temperature $T_{\mathrm{EM}}(x, y)$ defined as

$T_{\mathrm{EM}}(x, y)=\frac{\sum_{z} T(x, y, z) n_{\mathrm{e}}^{2}(x, y, z)}{\sum_{z} n_{\mathrm{e}}^{2}(x, y, z)}$,

and the temperature $T_{\mathrm{DEM}}(x, y)$ corresponding to the peak of the differential emission measure (DEM), defined as

$\operatorname{DEM}(T)=n_{\mathrm{e}}^{2} \frac{\mathrm{d} z}{\mathrm{~d} T}$

The results are summarized in Fig. 9. From this figure, it is clear that the average temperature $T_{\text {avg }}$ and the EM-weighted temperature $T_{\mathrm{EM}}$ are lower limits to the diagnosed temperature $T(C I F R)$, while the $T(C I F R)$ is never higher than the actual maximum temperature $T_{\max }$ encountered along the line of sight $l \equiv z$. The $T_{\text {DEM }}$ is not a good characteristic of the temperature structure along the line of sight, since it can be strongly biased towards cooler and denser voxels. The vertical strips in Fig. 9 bottom are caused by the chosen step in $\mathrm{d}\left(\log _{10} T\right)$, which we define to be equal to 0.02 dex.

A useful approximation of the diagnosed temperature $T(C I F R)$, according to Fig. 9 middle right, is the geometric mean of $T_{\mathrm{EM}}$ and $T_{\max }$, i.e.,

$T(C I F R) \approx \sqrt{T_{\mathrm{EM}} T_{\max }}$.

We note that the $T(C I F R)$ was derived under the assumption that the plasma is isothermal, which is clearly not true in the model. However, Eq. (24) allows one to connect the diagnosed $T(C I F R)$ to the thermal structure $T(z)$ along the line of sight, even if the $T(z)$ cannot be determined from the observations. It also shows that for non-isothermal plasma, $T(C I F R)$ should not be taken as a simple average (Reale et al. 2007) but is more biased towards the maximum value.

\section{Discussion}

\subsection{Comparison with other emission models}

We built a simple model of the active region corona using a steady heating, potential approximation of the magnetic field and the static energy equilibrium along a given field line. The static energy equilibrium is described by the scaling laws and the analytical $T(s)$ and $p(s)$ profiles (Sect. 2.2). Some authors used a similar approach (Schrijver \& Aschwanden 2002; Schrijver et al. 2004), while others used direct numerical modeling, which were restricted to the stable solutions (Warren \& Winebarger 2006; Lundquist et al. 2008a,b), included time-dependent heating (e.g., Warren \& Winebarger 2007), or built a full MHD model (Gudiksen \& Nordlund 2005a,b; Mok et al. 2008). None of these models were entirely successful in reproducing, or even attempting to reproduce, the observed emission simultaneously in both EUV and X-ray filters. The difference between these models and ours is that we explicitly included thermally unstable loops with very short heating scale-lengths by approximating their time-averaged state. This inclusion was done by extrapolating the analytical formulae describing the temperature and 

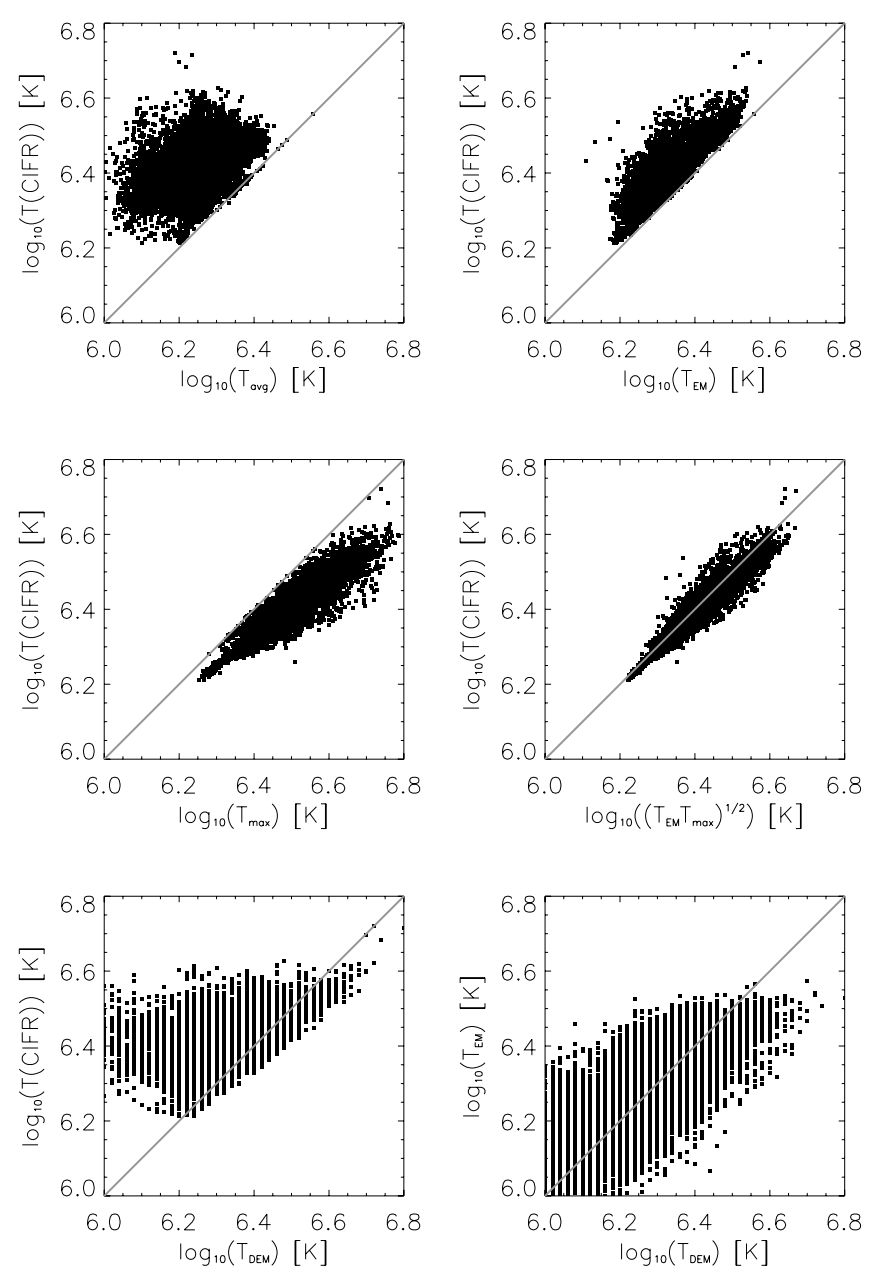

Fig. 9. Scatterplots of the dependence of the diagnosed $T(C I F R)$ on the characteristics of the temperature distribution in the best-fit model. These include $T_{\text {avg }}$ (top left), $T_{\mathrm{EM}}$ (top right), $T_{\max }$ (middle left), $\sqrt{T_{\mathrm{EM}} T_{\max }}$ (middle right), and $T_{\mathrm{DEM}}$ (bottom left). The dependence of $T_{\mathrm{EM}}$ on $T_{\mathrm{DEM}}$ is also plotted at the bottom right.

density. We also considered the spatially variable heating scalelength $s_{\mathrm{H}}^{\Gamma}$, which includes the loop expansion factor $\Gamma$ (Sect. 5.2).

As we showed in Sect. 4, this allowed us to built a model that does a reasonable job in reproducing the observed emission distributions in both the EUV and X-ray filters. In particular, our emission model matches both the spatial extent of the $\mathrm{X}$-ray core and the general emission distribution (intensity histograms), and contains coronal loops in all filters (Fig. 4). It also contains the moss and the secondary dipole, and matches the temperature structure derived from X-ray observations using the CIFR method (Sect. 4.1). The moss emission in the predicted EUV images is not as bright as in other models (Schrijver et al. 2004; Warren \& Winebarger 2006, 2007; Mok et al. 2005, 2008). The level of agreement between the observed and predicted $\mathrm{X}$-ray intensities, as described by the correlation coefficient $r$ and weighted-rms relative error $w$ (Eq. (21)), is in our model slightly better than in the model of Lundquist et al. (2008a). However, a detailed, direct pixel-to-pixel comparison does not provide good agreement especially for the X-ray filters, because of our potential extrapolation (Sect. 3.1.1).

We note that Mok et al. (2008) utilized a heating function that is locally dependent on the magnetic field. Their model
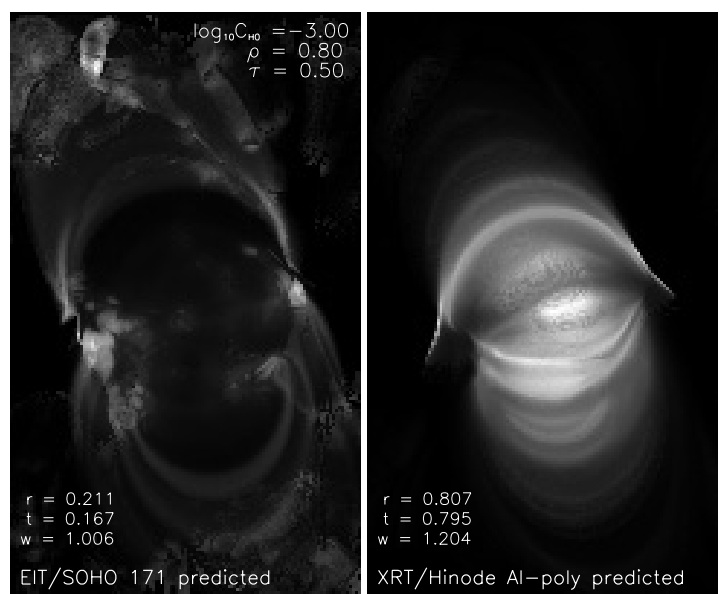

Fig. 10. Resulting model of emission without taking into account the loop expansion factor $\Gamma$. Left: predicted EIT 171 emission. Right: predicted XRT Al-poly emission showing strong sidelobes.

contains several, evolving EUV coronal loops. In accordance with observations, these individual EUV loops have nearly uniform cross-section and a high contrast. Their emission is also roughly consistent with observations. In our model, the true structure of the magnetic field is approximated by the parameters $B_{0}, L_{0}, s_{\mathrm{H}}$, and $\Gamma$.

\subsection{Loop expansion}

The loop expansion factor $\Gamma$ is an essential part of our model. Without taking into account its effect on $s_{\mathrm{H}}^{\Gamma}$ (Eq. (16)), the resulting emission would be very different (Fig. 10). In this figure, the modeled emission encompasses a larger area than the observations and contains a strong, unstructured diffuse component and sidelobes that are prominent in the XRT filters. These sidelobes are due to magnetic field lines rooted in the negativepolarity sunspot, where the field $B_{0}$ is at its strongest. The right sidelobe is located directly above this sunspot, at a location without any significant observed XRT signal. Sidelobes appear in the majority of models with widely different heating parameters. No combination of $C_{\mathrm{H} 0}, \rho$, and $\tau$ can reproduce the observed emission. We thus conclude that the loop expansion factor $\Gamma$ must be taken into account when modeling the active region coronal emission.

The emitting volume in the best-fit model in Fig. 4 corresponds to the volume where $\Gamma \lesssim 50$ (Fig. 11). On entering the quasiseparatrix layers (Priest \& Démoulin 1995; Démoulin et al. 1996; Titov et al. 2002), the value of $\Gamma$ increases rapidly to $\approx 10^{4}$. Such high values of $\Gamma$ then lead to high values of $L_{0} / s_{H}^{\Gamma}$ and prohibits the computation of $T$ and $n_{\mathrm{e}}$ in these voxels (Sect. 2.3). Thus, the emitting volume in the model is topologically confined between the quasiseparatrix layers and the chromosphere. A similar result was noted by Wang et al. (2000) for X-ray observations. The intersection of the quasiseparatrix layers with the photosphere (quasiseparatrices) are shown in Fig. 11 as thick green lines.

Schrijver et al. (2010) studied the connections between loop fans consisting of both open and closed loops observed by TRACE 171 filter and concluded that loop fans lie directly above the quasiseparatrices (the intersections of the quasiseparatrix layers with the photosphere). In light of our results, this means that $\Gamma>50$, at least for the closed coronal loops belonging to the fan. 
J. Dudík et al.: Is it possible to model observed active region coronal emission simultaneously in EUV and X-ray filters?

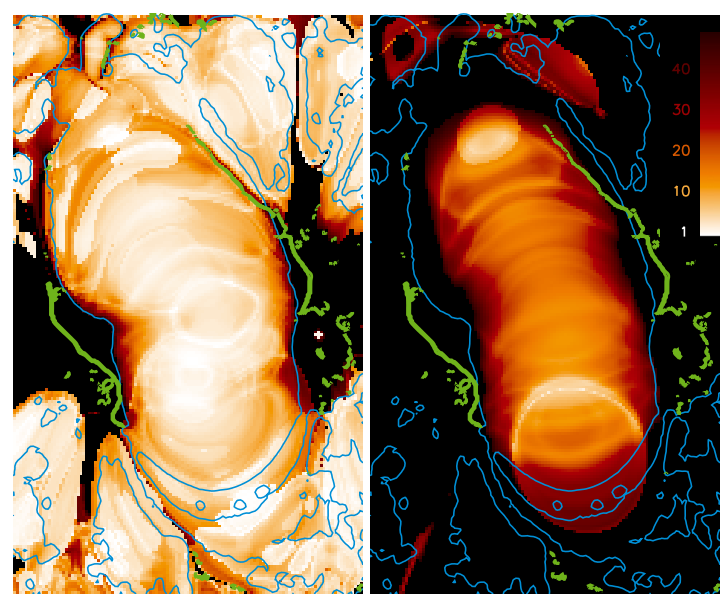

Fig. 11. Loop expansion factor $\Gamma$ at $z=2(3.6 \mathrm{Mm}$, left $)$ and $z=20$ (29.3 Mm, right) shown in inverse scale, saturated to value of $\Gamma=50$ (black color). Contour of $100 \mathrm{DN} \mathrm{s}^{-1} \mathrm{px}^{-1}$ of the predicted EIT 171 emission are plotted in cyan. Photospheric quasiseparatrices are shown as thick green lines.

Despite the loop expansion factor $\Gamma$, the apparent diameter of coronal loops in the predicted emission images (Fig. 4) does not exhibit significant expansion. However, the rate $s_{\mathrm{H}}$ of the magnetic field decrease along a loop, and the loop expansion factor $\Gamma$ (Fig. 11), both being the properties of the magnetic field, exhibit spatial variations on the scale given by the voxel size. Thus, the spatial variations in the $s_{\mathrm{H}}^{\Gamma}$ will also be on the scale of the voxel size, and finally, the spatial variations in the synthetic emission will be on the spatial scale of pixels. This explains the absence of significantly expanding loops in the predicted emission. We note that these spatial variations are probably resolution-dependent, although the study to confirm this is beyond the scope of this paper.

\subsection{Thermal nonequilibrium and its implications}

In Sect. 4.3, we found that the majority of the modeled warm loops, seen in the EIT 171 and 195 filters, have very short heating scale-lengths $s_{\mathrm{H}}^{\Gamma}$. These loops are known to be thermally unstable and should undergo the condensation-evaporation cycle (e.g., Müller et al. 2003, 2004, 2005; Karpen et al. 2005; Klimchuk et al. 2010). In our model, the cyclic solutions are incorporated only by using their average over evenly distributed phases (Sect. 2.4).

Ultimately, even with the spatially varying $s_{\mathrm{H}}^{\Gamma}$, we are unable to find a single model that simultaneously contains only stable loops and matches both the temperature structure and the EUV and X-ray observations in all filters (Sect. 4.1).

Klimchuk et al. (2010) studied whether thermally unstable loops, either monolithic or multi-strand, can match the observed ones. For monolithic loops, they obtained a highly structured intensity profile, a feature that is not observed. The intensity profile of multi-strand loops, averaged over the randomly distributed phases, was much more homogeneous. Nevertheless, they found that such loops would have lifetimes that are longer than the observed ones. In addition, the condensation-evaporation cycles in their model were only quasi-periodic, which presents a problem for the phasing of individual strands for more than one cycle. On the basis of these results, Klimchuk et al. (2010) ruled out thermal nonequilibrium, because of a too localized heating in the active regions, as an explanation of the warm 171 loops.
This poses a problem for our model. It means that even if our model does a reasonable job in reproducing the observations (Sects. 4.1, 5.1), and links this emission to the properties of the magnetic field (Sects. 2, 5.2), in the end it cannot explain all the observed features. That is, since our model must include unstable loops to match all observations, it is inherently not selfconsistent. The immediate implication is that the heating of at least the warm 171 and 195 loops should be dynamic, i.e., timedependent, since the possibilities of building emission models with static heating now seem to be exhausted.

Finally, we note that even if we exclude the unstable loops from the process of selection of the best-fit model in Sect. 4.1, the values of the heating parameters $C_{\mathrm{H} 0}, \rho$, and $\tau$ can still be valid. Excluding the unstable loops, a best-fit model must be found by using the EIT 284 and the X-ray filters only. The criterion of matching the temperature structure can obviously be kept. The criteria for the visual comparison can then be supplemented with for instance the requirement of matching the EIT 284 intensity histogram. Using these criteria, we arrive at the conclusion that only models with $\log _{10}\left(C_{\mathrm{H} 0}\right)=-3.0, \rho=0.7-$ 0.8 , and $\tau=0.5-0.75$ are suitable. These values are almost identical to those found in Sect. 4.1.

\subsection{Identification of the heating mechanism}

The purpose of building a model of active region coronal emission is to identify the heating mechanism by constraining the heating parameters. The proposed heating mechanisms were summarized and parametrized in Mandrini et al. (2000, Table 5 therein) and discussed and graphically summarized in Lundquist et al. (2008b, Table 1 and Fig. 12 therein).

At a glance, the parametrization of the best-fit model obtained in this paper, with $\rho=0.7-0.8$ and $\tau=0.5$, does not correspond to any of the proposed heating mechanisms. This is caused mainly by the parameter $\tau$, since the majority of the proposed coronal heating mechanisms have $\tau \leq 0$ or $\tau \geq 1$, with no models in-between. Although this interesting result does not enable us to identify the one (and only) mechanism heating the corona, it does not rule out the possibility that the corona is heated by multiple mechanisms.

We note that despite the majority of coronal heating models exhibiting a dependence on $B$ with $\rho$ equal to 1 or 2 , our result of $\rho=0.7-0.8$ is not in contradiction with some of these models. This can be shown by considering that the mass density $\rho_{m}$ is proportional to $p_{0} / T_{1}$, i.e., according to Eqs. (9) and (10)

$\rho_{m} \sim B_{0}^{4 \rho / 7} L_{0}^{(1-4 \tau) / 7}$

The heating by reconnection (model 4 ), based on the work of Parker (1983), scales as $B^{1} L^{-2} \rho_{m}^{1 / 2} V^{2} R^{1}$ (Mandrini et al. 2000), where $V$ is velocity and $R$ is the flux-tube radius. Using Eq. (25) and considering that $R \sim B^{-1 / 2}$ because of flux conservation (Eq. (15)), the model 4 scales as $B^{1 / 2+2 \rho / 7}$. This scaling has to be equal to $B^{\rho}$ implying that $\rho=0.7$, which is what we found in this paper. A similar result can be obtained for model 21 , where $\rho=5 / 7 \approx 0.714$, or for model 11 , where $\rho=0.875$.

The result of $\rho=0.7-0.8$ obtained in this paper agrees with the result of Fludra \& Ireland (2008), who found that $E_{\mathrm{H}} \sim \phi^{\gamma}$ by analyzing the line intensities observed by $\mathrm{CDS} / \mathrm{SOHO}$. In this relationship $\phi$, is the magnetic flux density at the footpoints of individual loops and $\gamma \in\langle 0.6,1.1\rangle$.

The scaling with $L$ cannot be as easily brought into agreement with $\tau=0.5$, leaving room for additional work. In our 
model, the higher the value of $\tau$, the more the emission is concentrated near the transition region. For $\tau=1$, the majority of the emission lies at very low altitudes, and very little emission comes from coronal altitudes (almost no apparent loops) for $\tau=2$. In our model, both the moss and the secondary dipole provide quite strong constrains on the value of $\tau$ (Sect. 4.1).

We point out that our scaling relationship obtained for heating, $E_{\mathrm{H}}=10^{-3}\left(B_{0} / B_{\mathrm{ref}}\right)^{0.7-0.8}\left(L_{\mathrm{ref}} / L_{0}\right)^{0.5} \exp \left(-\left(s-s_{0}\right) / s_{\mathrm{H}}^{\Gamma}\right)$ is in agreement with the results of Schrijver \& Aschwanden (2002) and also Schrijver et al. (2004), who as a result of their models found that the heating flux density scales as

$F_{\mathrm{H}}=2 \times 10^{4}\left(B_{0} / 100\right)^{1.0 \pm 0.5}\left(L_{0} / 24\right)^{-0.7 \pm 0.3}(V / 0.4)^{0.0 \pm 0.5}$

and

$F_{\mathrm{H}}=4 \times 10^{11} B_{0}^{1.0 \pm 0.3} / L_{0}^{1.0 \pm 0.5}$,

[W m $\left.{ }^{-2}\right]$ respectively. In our model, the heating flux $F_{\mathrm{H}}$ can be obtained by integrating Eq. (13) with respect to $z$, taking into account the vertical decrease of magnetic field $B(z)$, as

$$
\begin{aligned}
F_{\mathrm{H}} & =\int_{z_{0}}^{\infty} E_{\mathrm{H} 0} \exp \left(-z / z_{\mathrm{H}}\right)=z_{\mathrm{H}} E_{\mathrm{H} 0} \exp \left(-z_{0} / z_{\mathrm{H}}\right) \\
& \approx 1.7 \times 10^{4}\left(B_{0} / B_{\mathrm{ref}}\right)^{0.7-0.8}\left(L_{\mathrm{ref}} / L_{0}\right)^{0.5}
\end{aligned}
$$

for $z_{0}=3 \mathrm{Mm}$ and $z_{\mathrm{H}} \approx 20 \mathrm{Mm}$. The values of $\rho$ and $\tau$ obtained here are within the best-fit limits of Schrijver \& Aschwanden (2002) and Schrijver et al. (2004).

Rewriting Eqs. (26) and (27) using the values of $B_{\text {ref }}=100 \mathrm{G}$ and $L_{\text {ref }}=100 \mathrm{Mm}$ gives the value of constants as $7.4 \times 10^{3}$ and $4 \times 10^{3} \mathrm{~W} \mathrm{~m}^{-2}$, respectively. Aschwanden (2005, p. 357) estimate the flux $F_{\mathrm{H}}$ needed to heat the hydrostatic active region corona as $5 \times 10^{3} \mathrm{~W} \mathrm{~m}^{-2}$. Our result, $1.7 \times 10^{4} \mathrm{~W} \mathrm{~m}^{-2}$, is approximately three times higher. This value is required to match the modeled temperature distribution (Fig. 5 first column) to the observed one.

Finally, we must caution against attempts to derive scaling relationships of the form $p \sim L_{0}^{\beta}$ and $T \sim L_{0}^{\gamma}$ from observations as done in e.g. Porter \& Klimchuk (1995). Using their weighted $t$-statistic test on values obtained in the best-fit model (Sect. 4.1), we found that although these scaling relations are found to exist and be well defined, both $\beta$ and $\gamma$ are functions of the height $z$. Their values decrease approximately linearly from $\beta=-0.3$ and $\gamma=-0.2$ for $z=2$, corresponding to the height of $(z+1 / 2) \Delta z=3.6 \mathrm{Mm}$ (Sect. 2.2), to $\beta=-3.0$ and $\gamma=-1.1$ for $z=20$, corresponding to the height of $29.3 \mathrm{Mm}$. The errors $\Delta \beta$ and $\Delta \gamma$ are smaller than \pm 0.1 . The values of $\beta$ and $\gamma$ found from observations would then be some mixture of the real values depending on the visibility of coronal loops and their altitudes. In particular, the result that $\beta \approx 1$ and $\gamma \approx 0$ of Porter \& Klimchuk (1995) was interpreted as $E_{\mathrm{H}} \sim L_{0}^{-2}$ according to the scaling laws of Rosner et al. (1978), which correspond to Eqs. (9) and (10) in the limits $s_{\mathrm{H}} \rightarrow \infty$ and $z_{1} \ll \lambda_{p}\left(z_{1}\right)$. The presence of $s_{\mathrm{H}}$ and $\lambda_{p}$ in Eqs. (9) and (10) causes the departure of the $T_{1}\left(L_{0}\right)$ and $p_{0}\left(L_{0}\right)$ relations from the ones derived by Rosner et al. (1978) in such a way that a given $T_{1}$ or $p_{0}$ can be obtained for different $L_{0}$, with appropriate $s_{\mathrm{H}}$ and $\lambda_{p}$, even if $E_{\mathrm{H}} \nsim L_{0}^{-2}$. Thus, finding the power law between the observed loop lengths and their temperatures or densities is probably not a suitable way of discerning the properties of the coronal heating mechanism.

\section{Conclusions}

We have developed a model of the emission of the active region corona in three EUV and multiple X-ray filters. The model assumes that steady, non-uniform heating occurs at the footpoints. We included the spatially variable loop expansion factor $\Gamma$ and heating scale-length $s_{\mathrm{H}}^{\Gamma}$, and explored a wide range of heating function parameters. Our main findings can be summarized as follows:

1. The temperature structure of the active region corona plays a critical role in the modeling because of the strong dependence of the filter responses on $T$. In this paper, we have derived the temperature structure from X-ray observations of XRT/Hinode using the combined improved filter-ratio (CIFR) method. We have found that only a model whose temperature structure matches the temperature structure derived from observations can produce synthetic emission that approximates the observations. In our model, the temperature structure is directly linked to the spatial properties of the heating function. To match the observations, the model must include the spatially variable loop expansion factor $\Gamma$ and the heating scale-length $s_{\mathrm{H}}^{\Gamma}$, which are the properties of the magnetic field.

2. The best-fit model matches the general properties of the observed emission in all filters. In particular, it predicts coronal loops in both EUV and X-ray filters. However, the modeled loops appearing in the EIT/SOHO 171 and 195 filters are dominated by very short heating scale-lengths $s_{\mathrm{H}}^{\Gamma}$. These loops correspond to thermally unstable solutions that undergo the condensation-evaporation cycle. In our model, these unstable solutions are incorporated as the average over evenly distributed phases. With our steady heating, we were unable to find a model that does not contain the unstable loops and simultaneously matches observations in all filters.

3. Since our model cannot fit observations in all filters without the unstable solutions, it ultimately cannot lead to a selfconsistent result. This is because the lifetimes of such unstable loops would exceed the observed ones (Klimchuk et al. 2010). Since there do not seem to be any other possibilities of building emission models with steady heating, we conclude that, at least for the warm loops observed in the 171 and 195 filters, the heating should be dynamic.

4. The stable loops with sufficiently large $s_{\mathrm{H}}^{\Gamma}$ correspond to hot loops observed in the 284 filter and the X-ray filters. In our model, such large $s_{\mathrm{H}}^{\Gamma}$ are found in the active region core, but outside the sunspot umbrae, in accordance with the observed locations of the bright X-ray emission in the active regions.

5. The temperature $T(C I F R)$ derived using the CIFR method can be approximated by the geometric mean of the maximum temperature $T_{\max }$ and emission-measure-weighted temperature $T_{\mathrm{EM}}$ (Eq. (24)) along the line of sight.

6. The instrumental point-spread function should be subtracted from observations prior to their interpretation and modeling, since the presence of scattered light influences the relative intensities of various observed structures.

We conclude that despite our model based on steady heating being able to match the general properties of the emission and link them to the properties of the magnetic field, it does not provide self-consistent results. Thus, there is no clear answer to the question posed in the title. If there is a universal heating function, it should at least be partially time-dependent.

Acknowledgements. The authors thank the anonymous referee for remarks that led to improvements of the manuscript. This work was supported by Scientific 
Grant Agency, VEGA, Slovakia, Grant Nos. 1/0240/11, Grant IAA300030701 of the Grant Agency of the Academy of Sciences of the Czech Republic, and Grants Nos. 205/09/1705 and 205/09/1469 of the Grant Agency of the Czech Republic. J.D. acknowledges support from Comenius University grants No. UK/398/2009, UK/237/2010, and UK/57/2011. The author team is thankful to Dr. Markus J. Aschwanden and Dr. James A. Klimchuk for including their numerical codes into the SolarSoft library. During creation of Fig. 8, the "exponent" IDL function created by Stein V. H. Haugan was used. The Solar and Heliospheric Observatory $(\mathrm{SOHO})$ is a project of international cooperation between ESA and NASA. The Transition Region and Coronal Explorer (TRACE) is a mission of the Stanford-Lockheed Institute for Space Research, and part of the NASA Small Explorer program. Hinode is a Japanese mission developed and launched by ISAS/JAXA, with NAOJ as domestic partner and NASA and STFC (UK) as international partners. It is operated by these agencies in co-operation with ESA and NSC (Norway). CHIANTI is a collaborative project involving the NRL (USA), the Universities of Florence (Italy) and Cambridge (UK), and George Mason University (USA).

\section{References}

Alexander, D., Gary, G. A., \& Thompson, B. J. 1997, ASP Conf. Ser., 155, 100 Alissandrakis, C. E. 1981, A\&A, 100, 197

Antiochos, S. K., Karpen, J. T., DeLuca, E. E., Golub, L., \& Hamilton, P. 2003, ApJ, 590, 547

Aschwanden, M. J. 2005, Physics of the Solar Corona: An Introduction (Chichester, United Kingdom: Praxis Publishing Ltd.)

Aschwanden, M. J., \& Sandman, A. W. 2010, AJ, 140, 723

Aschwanden, M. J., \& Schrijver, C. J. 2002, ApJS, 142, 269

Aschwanden, M. J., Newmark, J. S., Delaboudinière, J.-P., et al. 1999, ApJ, 515, 842

Aschwanden, M. J., Alexander, D., Hulburt, N., et al. 2000, ApJ, 531, 1129

Aschwanden, M. J., Schrijver, C. J., \& Alexander, D. 2001, ApJ, 550, 1036

Aschwanden, M. J., Nitta, N. V., Wuelser, J.-P., \& Lemen J. R. 2008, ApJ, 680, 1477

Aschwanden, M. J., Wuelser, J. P., Nitta, N. V., Lemen, J. R., \& Sandman, A. W. 2009, ApJ, 695, 12

Avrett, E. H., \& Loeser, R. 2008, ApJS, 175, 229

Berger, T., De Pontieu, B., Schrijver, C. J., \& Title, A. M. 1999, ApJ, 519, 97

Brooks, D. H., \& Warren, H. P. 2009, ApJ, 703, L10

Brooks, D. H., Ugarte-Urra, I., \& Warren, H. P. 2008, ApJ, 689, 77

Cirtain, J. W., Del Zanna, G., DeLuca, E. E., et al. 2007, ApJ, 655, 598

Culhane, J. L., Harra, L. K., \& James, A. M. 2007, Sol. Phys., 243, 19

Defise, J.-M., Clette, F., Moses, J. D., \& Hochedez, J.-F. E. 1997, in Proc. EUV, X-ray and Gamma-Ray Instrumentation for Astronomy VIII, ed. O. H. Sigmund, \& M. A. Gummin, SPIE, 3114, 598

DeForest, C. E. 2007, ApJ, 661, 532

DeForest, C. E., Martens, P. C. H., \& Wills-Dawey, M. J. 2009, ApJ, 690, 1264

Delaboudinière, J.-P., Artzner, G. E., Brunaud, J., et al. 1995, Sol. Phys., 162, 291

Démoulin, P., Hénoux, J. C., Priest, E. R., \& Mandrini, C. H. 1996, A\&A, 308, 643

Dere, K. P, Landi, E., Mason, H. E., Monsignori Fossi, B. C., \& Young, P. R. 1997, A\&AS, 125, 149

DeRosa, M. L., Schrijver, C. J., Barnes, G., et al. 2009, ApJ, 696, 1780

Domingo, V., Fleck, B., \& Poland, A. I. 1995, Sol. Phys., 162, 1

Dudík, J., Dzif̌ćáková, E., Karlický, M., \& Kulinová, A. 2009a, A\&A, 502, 957

Dudík, J., Kulinová, A., Dzifč́áková, E., \& Karlický, M. 2009b, A\&A, 505, 1255

Fletcher, L., \& De Pontieu, B. 1999, ApJ, 520, 135

Fludra, A., \& Ireland J. 2008, A\&A, 483, 609

Freeland, S. N., \& Handy, B. N. 1998, Sol. Phys., 182, 497

Gary, G. A. 1989, ApJS, 69, 323

Golub, L., Deluca, E., Austin, G., et al. 2007, Sol. Phys., 243, 63

Gontikakis, C., Contopoulos, I., \& Dara, H. C. 2008, A\&A, 489, 441

Gudiksen, B. V., \& Nordlund, Å. 2005a, ApJ, 618, 1020

Gudiksen, B. V., \& Nordlund, A.. 2005b, ApJ, 618, 1031

Handy, B. N., Acton, L. W., Kankelborg, C. C., et al. 1999, Sol. Phys., 187, 229

Kano, R., Sakao, T., Hara, H., et al. 2008, Sol. Phys., 249, 263

Karpen, J. T., Tanner, S. E. M., Antiochos, S. K., \& DeVore, C. R. 2005, ApJ, 635,1319

Karpen, J. T., Antiochos, S. K., \& Klimchuk, J. A. 2006, ApJ, 637, 531

Klimchuk, J. A. 2000, Sol. Phys., 193, 53
Klimchuk, J. A. 2006, Sol. Phys., 234, 41

Klimchuk, J. A., Karpen, J. T., \& Antiochos, S. K. 2010, ApJ, 714, 1239

Kosugi, T., Matsuzaki, K., Sakao, T., et al. 2007, Sol. Phys., 234, 3

Kuin, N. P. M., \& Martens, P. C. H. 1982, A\&A, 108, L1

Landi, E., Del Zanna, G., Dere, K. P., Mason, H. E., \& Landini, M. 2006, ApJS, 162,261

López Fuentes, M. C., Klimchuk, J. A., \& Démoulin, P. 2006, ApJ, 639, 459 López Fuentes, M. C., Démoulin, P., \& Klimchuk, J. A. 2008, ApJ, 673, 586 Lundquist, L. L., Fisher, G. H., \& McTiernan, J. M. 2008a, ApJS, 179, 509 Lundquist, L. L., Fisher, G. H., Metcalf, T. R., Leka, K. D., \& McTiernan, J. M. 2008b, ApJ, 689, 1388

Mandrini, C. H., Démoulin, P., \& Klimchuk, J. A. 2000, ApJ, 530, 999

Martens, P. C. H. 2010, ApJ, 714, 1290

Martens, P. C. H., Kankelborg, C. C., \& Berger, T. E. 2000, ApJ, 537, 471

Mok, Y., Mikić, Z., Lionello, R., \& Linker, J. A. 2005, ApJ, 621, 1098

Mok, Y., Mikić, Z., Lionello, R., \& Linker, J. A. 2008, ApJ, 769, 161

Müller, D. A. N., Hansteen, V. H., \& Peter, H. 2003, A\&A, 441, 605

Müller, D. A. N., Peter, H., \& Hansteen, V. H. 2004, A\&A, 424, 289

Müller, D. A. N., DeGroof, A., Hansteen, V. H., \& Peter, H. 2005, A\&A, 436, 1067

O’Dwyer, B., Del Zanna, G., Mason, H. E., et al. 2010, A\&A, 525, A137

Parenti, S., Reale, F., \& Reeves, K. K. 2010, A\&A, 517, A41

Parker, E. N. 1983, ApJ, 264, 642

Peres, G., Reale, F., \& Golub, L. 1994, ApJ, 422, 412

Porter, L. J., \& Klimchuk, J. A. 1995, ApJ, 454, 499

Priest, E. R., \& Démoulin, P. 1995, J. Geophys. Res., 100, A12, 23443

Reale, F., Parenti, S., Reeves, K. K., et al. 2007, Science, 318, 1582

Reale, F., Testa, P., Klimchuk, J. A., \& Parenti, S. 2009a, ApJ, 698, 756

Reale, F., McTiernan, J. M., \& Testa, P. 2009b, ApJ, 704, L56

Rosner, R., Tucker, W. H., \& Vaiana, G. S. 1978, ApJ, 220, 643

Sandman, A. W., Aschwanden, M. J., DeRosa, M. L., Wülser, J. P., \& Alexander, D. 2009, Sol. Phys., 259, 1

Scherrer, P. H., Bogart, R. S., Bush, R. I., et al. 1995, Sol. Phys., 162, 129

Schmelz, J. T., \& Martens, P. C. H. 2006, ApJ, 636, L49

Schmelz, J. T., Nasraoui, K., Richardson, V. L., et al. 2005, ApJ, 627, L81

Schmelz, J. T., Nasraoui, K., Del Zanna, G., et al. 2007a, ApJ, 658, L119

Schmelz, J. T., Kashyap, V. L., \& Weber, M. A. 2007b, ApJ, 660, L157

Schmelz, J. T., Scott, J., \& Rightmire, L. A. 2008, ApJ, 684, L115

Schmelz, J. T., Nasraoui, K., Rightmire, L. A., et al. 2009a, ApJ, 691, 503

Schmelz, J., Saar, S. H., DeLuca, E. E., et al. 2009b, ApJ, 693, L131

Schmelz, J., Kashyap, V. L., Saar, S. H., et al. 2009c, ApJ, 704, 863

Schrijver, C. J., \& Aschwanden, M. J. 2002, ApJ, 566, 1147

Schrijver, C. J., Title, M. A., Berger, T. E., et al. 1999, Sol. Phys., 187, 261

Schrijver, C. J., Sandman, A. W., Aschwanden, M. J., \& DeRosa, M. L. 2004, ApJ, 615, 512

Schrijver, C. J., DeRosa, M. L., \& Title, A. M. 2010, ApJ, 719, 1083

Serio, S., Peres, G., Vaiana, G. S., et al. 1981, ApJ, 243, 288

Spitzer, L. 1962, Physics of Fully Ionized Gases (New York: Interscience)

Susino, R., Lanzafame, A. C., Lanza, A. F., \& Spadaro, D. 2010, ApJ, 709, 499

Terzo, S., \& Reale, F. 2010, A\&A, 515, A7

Titov, V. S., Hornig, G., \& Démoulin, P. 2002, J. Geophys. Res., 108, A8, 1164

Tripathi, D., Mason, H. E., Dwivedi, B. N., del Zanna, G., \& Young, P. R. 2009, ApJ, 694, 1256

Tripathi, D., Mason, H. E., del Zanna, G., \& Young, P. R. 2010, A\&A, 518, A42

Ugarte-Urra, I., Winebarger, A. R., \& Warren, H. P. 2006, ApJ, 643, 1245

Ugarte-Urra, I., Warren, H. P., \& Brooks, D. H. 2009, ApJ, 695, 642

Vásquez, A. M., Frazin, R. A., \& Kalamabadi, F. 2009, Sol. Phys., 256, 73

Wang, H., Yan, Y., Sakurai, T., \& Zhang, M. 2000, Sol. Phys., 197, 263

Warren, H. P., \& Winebarger, A. R. 2006, ApJ, 645, 711

Warren, H. P., \& Winebarger, A. R. 2007, ApJ, 666, 1245

Warren, H. P., Winebarger, A. R., Mariska, J. T., Doschek, G. A., \& Hara, H. 2008, ApJ, 677, 1395

Warren, H. P., Winebarger, A. R., \& Brooks, D. H. 2010, ApJ, 711, 228

Watko, J. A., \& Klimchuk, J. A. 2000, Sol. Phys., 193, 77

Weber, M. A., Schmelz, J. T., DeLuca, E. E., \& Roames, J. K. 2005, ApJ, 635, L101

Winebarger, A. R., Warren, H. P., \& Mariska, J. T. 2003, ApJ, 587, 439

Winebarger, A. R., Warren, H. P., \& Falconer, D. 2008, ApJ, 676, 672

Vernazza, J. E., Avrett, E. H., \& Loeser, R. 1981, ApJ, 45, 635

Wülser, J. P., Lemen, J. R., Tarbell, T. D., et al. 2004, Proc. Int. Soc. Opt. Eng., 5171, 111 